Mens

Revue d'histoire intellectuelle et culturelle

mens

\title{
De Montmartre à Broadway : le cabaret Au matou botté de Montréal (1929-1932)
}

\section{Luc Bellemare}

Volume 16, numéro 1, automne 2015

Vie musicale amateur, populaire et américaine à Montréal, 1918-1958

URI : https://id.erudit.org/iderudit/1038984ar

DOI : https://doi.org/10.7202/1038984ar

Aller au sommaire du numéro

Éditeur(s)

Centre de recherche en civilisation canadienne-française

ISSN

1492-8647 (imprimé)

1927-9299 (numérique)

Découvrir la revue

Citer cet article

Bellemare, L. (2015). De Montmartre à Broadway : le cabaret Au matou botté de Montréal (1929-1932). Mens, 16(1), 63-105. https://doi.org/10.7202/1038984ar

\section{Résumé de l'article}

L'existence éphémère du cabaret Au matou botté (1929-1932) de Montréal illustre à merveille la " crise de l'homme et de l'esprit » entrant dans la modernité, telle que décrite par l'historien Yvan Lamonde. En novembre 1929, les revuistes Roméo Poirier et Albéric Bourgeois fondent le cabaret Au matou botté à l'étage du restaurant Kerhulu \& Odiau, dans le Quartier latin. Envers et contre tous les obstacles, ce café-concert va vivre trois grandes périodes créatives. De l'ouverture jusqu'à mai 1930, la boîte calquée sur le modèle de Montmartre connaît un vif succès en occupant une niche identitaire canadienne-française laissée vacante par les autres lieux de divertissement de la ville. La programmation inclut des chansons françaises, des récitals lyriques, des sketches comiques et des thés dansants. En mai 1930, lorsque Paul Max et Eugène Vivez reprennent la direction artistique, le cabaret adopte le style plus rentable d'un nightclub de Broadway, avec orchestre de jazz, chansons anglaises, pantomimes italiennes et animal dances - fox-trot et autres danses aux pas inspirés d'animaux. Les touristes apprécient bien l'ambiance renouvelée, mais la clientèle des fastes débuts a déserté. À l'automne 1930, le cabaret semble se chercher une identité basée sur un compromis entre Montmartre et Broadway. Nous verrons pourquoi l'organisation d'événements spéciaux, la présence d'artistes locaux et internationaux, la contribution de vedettes de cinéma et la diffusion d'une émission au poste radio CKAC ne suffiront malheureusement pas à assurer la viabilité des lieux.
Ce document est protégé par la loi sur le droit d'auteur. L'utilisation des services d'Érudit (y compris la reproduction) est assujettie à sa politique d'utilisation que vous pouvez consulter en ligne.

https://apropos.erudit.org/fr/usagers/politique-dutilisation/ 


\title{
De Montmartre à Broadway : le cabaret Au matou botté de Montréal (1929-1932) ${ }^{1}$
}

\author{
Luc Bellemare \\ Chercheur indépendant
}

\section{Résumé}

L'existence éphémère du cabaret Au matou botté (1929-1932) de Montréal illustre à merveille la " crise de l'homme et de l'esprit " entrant dans la modernité, telle que décrite par l'historien Yvan Lamonde. En novembre 1929, les revuistes Roméo Poirier et Albéric Bourgeois fondent le cabaret Au matou botté à l'étage du restaurant Kerhulu \& Odiau, dans le Quartier latin. Envers et contre tous les obstacles, ce café-concert va vivre trois grandes périodes créatives. De l'ouverture jusqu'à mai 1930, la boîte calquée sur le modèle de Montmartre connaît un vif succès en occupant une niche identitaire canadienne-française laissée vacante par les autres lieux de divertissement de la ville. La programmation inclut des chansons françaises, des récitals lyriques, des sketches comiques et des thés dansants. En mai 1930, lorsque Paul Max et Eugène Vivez reprennent la direction artistique, le cabaret adopte le style plus rentable d'un nightclub de Broadway, avec orchestre de jazz, chansons anglaises, pantomimes italiennes et animal dances - fox-trot et autres danses aux pas inspirés

${ }^{1}$ La rédaction de cet article a été rendue possible grâce à une bourse de recherche postdoctorale du Fonds de recherche du Québec - Société et Culture. Je tiens aussi à remercier mes collègues Sandria P. Bouliane et Peggy Roquigny de m'avoir communiqué des renseignements complémentaires. 
d'animaux. Les touristes apprécient bien l'ambiance renouvelée, mais la clientèle des fastes débuts a déserté. À l'automne 1930, le cabaret semble se chercher une identité basée sur un compromis entre Montmartre et Broadway. Nous verrons pourquoi l'organisation d'événements spéciaux, la présence d'artistes locaux et internationaux, la contribution de vedettes de cinéma et la diffusion d'une émission au poste radio CKAC ne suffiront malheureusement pas à assurer la viabilité des lieux.

\section{Abstract}

The short existence of the cabaret Au matou botté (1929-1932) in Montreal perfectly depicts the "crisis of man and spirit" engaging into modernity, as demonstrated by historian Yvan Lamonde. In November 1929, Roméo Poirier and Albéric Bourgeois launch the French cabaret Au matou botté on the second floor of the restaurant Kerhulu \& Odiau, in the Quartier Latin. Facing many difficulties, the place will run through three distinct stages of creation. From the opening day up to May 1930, the Montmartre café enjoys great success with its genuine French Canadian program, thus contrasting with all other entertainment places in town. This program includes French chansons, operettas, comedies and dancing teas. In May 1930, when Paul Max and Eugène Vivez take over the management, the cabaret turns into a Broadway nightclub, with a jazz orchestra, songs in English, pantomimes and animal dances - such as fox-trot and other animal dance steps. Whereas tourists appreciate the new mood, customers of the opening days have gone. In the fall of 1930, the cabaret looks for a compromise between Montmartre and Broadway. In spite of special events, local and international feature artists, movie theatre stars and a special CKAC radio broadcast, we shall see why the cabaret won't insure its viability.

Situons-nous au mois de novembre 1929, au tout début de la grande crise économique. Alors que Madame Bolduc (1894-1941) va bientôt connaître un succès tout à fait inattendu sur disque, le 
cabaret Au matou botté ouvre ses portes dans le Quartier latin de Montréal. À ce moment, le centre-ville abonde en théâtres anglais et en nightclubs façon Broadway. Le nouveau café-concert va s'en distinguer en s'affichant résolument à la mode de Montmartre, devenant pour un temps la seule salle de la métropole à se consacrer aux spectacles de chansons françaises. La direction artistique est d'abord assurée par Albéric Bourgeois (1876-1962) et Roméo Poirier (1893-?), deux revuistes de scène et " chansonniers ", au sens alors courant de paroliers de chansons d'actualité sur des airs connus. Afin de favoriser la profitabilité de l'entreprise, le cabaret Au matou botté loge à l'intérieur du restaurant Kerhulu \& Odiau, ce qui permet de jumeler le repas au divertissement. Or cela se révèle vite insuffisant. Les années de crise sont extrêmement difficiles pour les affaires de tout le monde. Comment peut-on concilier, à Montréal, le projet identitaire d'un cabaret de Montmartre avec une couleur canadienne forte et celui indéniablement plus rentable d'un cabaret de New York, dans la mouvance des nouveaux orchestres de danse, ou Big Bands de jazz? Cette dialectique, on le verra, illustre admirablement la " crise de l'homme et de l'esprit " entrant dans la modernité, telle que décrite par l'historien Yvan Lamonde ${ }^{2}$.

À Montréal, l'aventure éphémère du café-concert à l'étude précède de vingt ans celle du cabaret Au faisan doré (1947-1950) de Jacques Normand ${ }^{3}$, et celle, à Québec, du cabaret Chez Gérard (1948-1977) de Gérard Thibault ${ }^{4}$. On se situe longtemps avant l'ouverture de la Comédie-Canadienne (1958-1971) de Gratien Gélinas 5 et des premières grandes boîtes à chansons, Chez Bozo

2 Yvan Lamonde, La modernité au Québec : la crise de l'homme et de l'esprit, 19291939, Montréal, Éditions Fides, 2011.

3 Jacques Normand, Les nuits de Montréal, préface de Roger Baulu et Charles Aznavour, Montréal, Éditions La Presse, 1974.

4 Chantal Hébert et Gérard Thibault, Chez Gérard: la petite scène des grandes vedettes, 1938-1978, Sainte-Foy, Éditions spectaculaires, 1988.

5 Anne-Marie Sicotte, Gratien Gélinas : la ferveur et le doute, Montréal, Typo, 2009. 
(mai 1959) et La Butte à Mathieu (novembre 1959), par exemple ${ }^{6}$. L'histoire méconnue du cabaret Au matou botté sera relatée à partir du dépouillement exhaustif des communiqués, critiques et publicités parus dans les grands quotidiens de même que dans certaines revues culturelles de l'époque. Quelques fragments de programmes, d'iconographie et de feuilles de musique retrouvés dans les archives viendront étayer l'argumentation.

En guise d'introduction, je brosserai un bref portrait des nouveaux divertissements qui alimentent le café-concert à Montréal depuis 1900. Je présenterai ensuite un survol des revues créées par les maîtres d'œuvre Bourgeois et Poirier avant l'ouverture du cabaret Au matou botté. L'analyse des données me permettra enfin de déterminer trois grandes périodes de création : 1) celle du café-concert de Montmartre; 2) celle du nightclub de Broadway; et 3) celle du compromis impossible entre les pôles de Paris et de New York.

\section{Aperçu des divertissements à Montréal (1900-1930)}

De la Belle Époque à l'entre-deux-guerres, la ville de Montréal est inondée d'une pléthore de nouveaux divertissements importés d'Europe et d'ailleurs en Amérique du Nord : il y a l'opérette, la mélodie classique et la musique de salon, que l'on s'approprie dans les foyers grâce aux partitions en feuilles pour le piano ${ }^{7}$; les spectacles

${ }^{6}$ Daniel Guérard, La belle époque des boîtes à chansons, Montréal, Stanké, 1996; ainsi que Gilles Mathieu et Sylvain Rivière, La butte à Mathieu, Montréal, VLB éditeur, 2010.

7 Mireille Barrière, La société canadienne-française et le théâtre lyrique à Montréal entre 1840 et 1913, thèse de doctorat, Québec, Université Laval, 1990; Hugo Lévesque, La Société canadienne d'opérette (1921-1934) : premier jalon de l'émancipation de la scène lyrique au Québec, mémoire de maîtrise, Montréal, Université de Montréal, 2004; Micheline Cambron (dir.), La vie culturelle à Montréal vers 1900, Montréal, Éditions Fides, 2005; Marie-Thérèse Lefebvre et Jean-Pierre Pinson (dir.), Chronologie musicale du Québec, 1534-2004: musique de concert et musique religieuse, avec la collaboration de Mireille Barrière et al., Sillery, Éditions du Septentrion, 2009; ainsi que Mireille Barrière, "Les conditions de travail des artistes de la scène au Québec (1893-1914) : une profession en construction ", Revue d'histoire de l'Amérique française, vol. 67, n² (automne 2013), p. 137-163. 
au pavillon du parc Sohmer ${ }^{8}$; les restaurants, cabarets et salles avec orchestre de danse ${ }^{9}$; les vues animées du Ouimetoscope, relayées par le cinéma parlant au tournant des années $1930^{10}$; la presse à grand tirage et les nombreuses revues culturelles imprimées ${ }^{11}$; le gramophone d'Emile Berliner (1851-1929) et le disque plat, suivis par la radiodiffusion au poste $\mathrm{CKAC}^{12}$; le théâtre burlesque, les revues d'actualité, monologues comiques, sketches, comédies musicales et scènes folkloriques ${ }^{13}$. De Paris à New York, en passant

8 Fondé par Ernest Lavigne (1851-1909), le parc Sohmer de Montréal (1889-1919) présente une riche programmation de musique classique, marches militaires, romances et opérettes, cirque, danse, lutte et boxe (Yvan Lamonde et Raymond Montpetit, Le parc Sohmer de Montréal, 1889-1919: un lieu populaire de culture urbaine, Québec, Institut québécois de recherche sur la culture, 1986).

${ }^{9}$ Nancy Marrelli, Stepping Out: The Golden Age of Montreal Night Clubs, 1925-1955, Montréal, Véhicule Press, 2004; John Gilmore, Une histoire du jazz à Montréal, préface de Gilles Archambault, traduit de l'anglais par Karen Ricard, Montréal, Lux éditeur, 2009; Peggy Roquigny, Les plaisirs de la danse à Montréal : transformation d'un divertissement et de ses pratiques, 1870-1940, thèse de doctorat (histoire), Montréal, Université du Québec à Montréal, 2012.

10 Ouvert en 1906 par Léo-Ernest Ouimet (1877-1972), le Ouimetoscope est la première salle construite pour la projection du cinéma muet à Montréal. Voir Germain Lacasse, Histoire de scopes : le cinéma muet au Québec, Montréal, Cinémathèque québécoise, Musée du cinéma, 1988; ainsi que Germain Lacasse, Johanne Massé et Bethsabée Poirier, Le diable est en ville : Alexandre Silvio et l'émergence de la modernité populaire au Québec, Montréal, Les Presses de l'Université de Montréal, 2012.

11 Pour les journaux, j'ai consulté La Patrie, La Presse et le Montreal Daily Star. Pour les revues culturelles, j'ai dépouillé La Lyre, La Revue moderne et L'œil en coulisse. Les archives de ces publications proviennent des collections numériques, des microfilms et des fonds de Bibliothèque et Archives nationales du Québec (BAnQ), à Montréal.

12 Luc Bellemare, Les réseaux des "Lyriques" et des "Veillées»: une histoire de la chanson au Québec dans l'entre-deux-guerres par la radiodiffusion au poste CKAC de Montréal (1922-1939), thèse de doctorat, Québec, Université Laval, 2012; et Sandria P. Bouliane, "Good-bye Broadway, Hello Montréal " : traduction, appropriation et création de chansons populaires canadiennes-françaises dans les années 1920, thèse de doctorat, Québec, Université Laval, 2013.

13 Chantal Hébert, Le burlesque au Québec : un divertissement populaire, préface d'Yvon Deschamps, La Salle, Hurtubise HMH, 1981; André G. Bourassa et Jean-Marc Larrue, Les nuits de la "Main" : cent ans de spectacles sur le boulevard Saint-Laurent (1891-1991), Montréal, VLB éditeur, 1993; Marc Charpentier, Broadway North: 
par Montréal, les cafés-concerts et cabarets empruntent à presque tous ces divertissements.

Dans son ouvrage sur l'Opéra français de Montréal (1893-1896), l'historienne Mireille Barrière montre à quel point les frontières entre les genres de la musique vocale demeurent alors floues, tant dans la programmation des compagnies d'opéra que dans celle des troupes itinérantes, de passage dans la métropole canadienne :

[Aux États-Unis], on affublait de ce nom [prestigieux d'opéra] des spectacles aussi hétéroclites que des minstrel shows, des extravaganzas et des parodies d'opéras célèbres. Des troupes de burlesque, enrobées d'épithètes exotiques, comme "éthiopiennes » et même "lilliputiennes ", s'affichent sous la raison sociale d' " opera companies »! La confusion persiste jusque dans la dénomination des lieux. Si à Paris, par exemple, l'Opéra, théâtre d'État, diffuse principalement ce répertoire [classique], il n'en est pas de même aux États-Unis et au Canada où foisonnent les " opera houses " qui n'ont rien à voir avec ce genre très sérieux, sauf que, par ce biais, les propriétaires de théâtres visaient à leur conférer prestige et respectabilité aux yeux du public ${ }^{14}$.

Bien que cette imbrication de l'opéra et des variétés touche le caféconcert à Montréal au tout début du $\mathrm{xx}^{\mathrm{e}}$ siècle, on connaît encore mal le processus d'implantation du genre au Québec. Le Théâtre l'Eldorado (1899-v. 1901) est possiblement le premier établissement local à reprendre le modèle du cabaret Le Chat noir, ouvert à Montmartre en 1881 par Rodolphe Salis (1851-1897) ${ }^{15}$. L'histoire

Musical Theater in Montreal in the 1920s, thèse de doctorat, Montréal, Université McGill, 1999; Robert Aird, "De coutlée au stand up comique : l'évolution du monologue québécois, de 1900 à nos jours ", Globe : revue internationale d'études québécoises, vol. 11, n 2 (2008), p. 23-41; et Lucie Robert (dir.), Apprivoiser la modernité théatrale : la pièce en un acte de la Belle Époque à la Crise : anthologie, Montréal, Éditions Nota bene, 2012.

${ }^{14}$ Mireille Barrière, L’Opéra français de Montréal (1893-1896) : l'étonnante histoire d'un succès éphémère, Montréal, Éditions Fides, 2000, p. 94.

15 En ce qui concerne l'ouverture de l'Eldorado de Montréal par Alexandre Boiron, voir La Patrie, 11 mars 1899, p. 11. 
de ce lieu reste à écrire, mais pour ce que l'on en sait, la chanson, l'opérette, les scènes dramatiques et l'orchestre à danser s'y entremêlent volontiers. Quelques œuvres méconnues, comme la revuevaudeville Paris-Montréal de Lucien Boyer et Numa Blès ${ }^{16}$, témoignent pourtant des échanges culturels qui ont cours à cette époque. Ailleurs en ville, le Monument-National (ouvert vers 1893), le Théâtre National (ouvert en 1900), le Théâtre des Nouveautés (v. 1902-1908), le Chanteclerc (ouvert en 1912), le Saint-Denis (ouvert en 1915) et le Canadien-français (ouvert vers 1919) seraient les principales salles où l'auditoire francophone peut assister à des spectacles apparentés ${ }^{17}$.

Les chansons qui meublent les revues de scène, les monologues et les sketches dramatiques à caractère folklorique sont fréquemment composées sur des airs connus, que l'on nomme aussi " timbres". Ceux-ci sont tirés de mélodies du folklore, d'airs de chansons populaires en France, d'extraits de vaudevilles et d'airs d'opérette. De Pierre-Jean de Béranger (1780-1857) à Yvette Guilbert (18651944), on saisit bien là l'essence du répertoire des cafés-concerts montmartrois, en France comme au Québec. Retenons pour l'heure que le symbole du Chat noir et l'esprit canaille auquel il renvoie auront leur importance pour la suite de cette histoire du café-concert à Montréal. Avant d'aller plus loin, il paraît opportun de présenter les premières réalisations de Bourgeois et Poirier, futurs responsables de l'ouverture du cabaret Au matou botté.

${ }^{16}$ Lucien Boyer et Numa Blès, Paris-Montréal : revue-vaudeville en trois actes: couplets, chansons, rondeaux et chours, Montréal, Imprimerie La Patrie, 1902.

17 Il est également à noter que la chanson politique s'exprime dans la presse, les revues imprimées et la musique en feuilles. Le Chansonnier politique du canard (1879) de Rémi Tremblay (1847-1926) est un bon exemple de railleries des politiciens canadiens. Voir Rémi Tremblay, Aux chevaliers du noud coulant : poèmes et chansons, édition établie, annotée et présentée par Jean Levasseur, Québec, Les Presses de l'Université Laval, 2007. D'autres airs de cabarets parisiens paraissent au Québec dans la revue Le Passe-temps, de même qu'aux éditions Archambault, J.-E. Bélair et Albert Turcotte. 


\section{Albéric Bourgeois, Roméo Poirier et leurs revues des années 1920}

\section{La Soirée chatnoiresque (1924)}

Albéric Bourgeois est d'abord identifié à ses caricatures et chroniques publiées dans le journal La Presse entre 1905 et $1957^{18}$. Dans les deux cas, son travail met en scène le personnage comique de Baptiste Ladébauche, une sorte d'habitant canadien, observateur posant un regard à la fois ludique et caustique sur l'actualité nationale et internationale. Bourgeois est également actif à titre de revuiste et de parolier de chansons d'actualité, d'abord sur scène, puis à la radio de $\mathrm{CKAC}^{19}$.

En novembre 1924, un communiqué annonce la tenue prochaine à Montréal d'une Soirée chatnoiresque d'un genre tout à fait nouveau ${ }^{20}$. Albéric Bourgeois organise cet événement à la Salle des Artisans, conjointement avec le chanteur Charles Marchand (1890-1930), la diseuse Camille Bernard (1898-1984) et le pianiste d'accompagnement Ernest Patience. Le spectacle donne lieu à des dialogues sur des sujets d'actualité et à un programme de chansons de café-concert ${ }^{21}$. La scène s'ouvre alors qu'un artiste peintre (Albéric Bourgeois) s'affaire à réaliser le portrait en pochade d'un modèle féminin (Camille Bernard). Tandis que la dame déclame quelques vers de poésie, on voit intervenir le troisième personnage (Charles Marchand). Il sera tour à tour question de la politique canadienne d'Arthur "Titur " Meighen (1874-1960) et William Lyon « Mac » Mackenzie King

18 Léon-A. Robidoux (dir.), Albéric Bourgeois, caricaturiste, préface de Normand Hudon et Robert LaPalme, Montréal, VLB éditeur, 1978.

19 Luc Bellemare, "Baptiste Ladébauche, du folklore au cabaret : à propos des collaborations d'Albéric Bourgeois et de Charles Marchand dans les années 1920 ", dans Micheline Cambron et Dominic Hardy (dir.), Quand la caricature sort du journal: Baptiste Ladébauche (1878-1957), Montréal, Éditions Fides, 2015.

20 La Presse, 15 novembre 1924, p. 38; et La Presse, 6 décembre 1924, p. 52-53.

${ }^{21}$ Le Fonds Albéric-Bourgeois (MSS346) de Bibliothèque et Archives nationales du Québec (ci-après BAnQ) Vieux-Montréal contient un texte de quatre pages dactylographiées qui m’a permis de reconstituer l'œuvre. 
(1874-1950), de la construction du pont de Longueuil - le pont Jacques-Cartier, inauguré en 1930 -, de la prohibition de l'alcool et des coiffures à la garçonne chez les jeunes femmes. Cette Soirée chatnoiresque est la première trace d'une collaboration artistique entre Bourgeois et Marchand ${ }^{22}$.

Police... en... quête? (1925)

Quant à Roméo Poirier, avocat de formation, on sait qu'il s'adonne au théâtre et à la musique. Du 2 au 8 mars 1925, il présente au Théâtre Orpheum la revue d'actualité Police... en... quête?, sa toute première création pour la scène ${ }^{23}$. Henri Letondal (1901-1955) note, dans La Patrie et dans La Lyre, que Poirier est un nouveau venu dans le monde dramatique. Inexpérimentée dans ce genre d'écriture, sa plume ne manque pourtant ni de gaieté ni de traits spirituels. Le public, composé de juges, d'avocats, d'échevins, de politiciens et de policiers qui ne craignent pas l'autodérision, remplit aux quatre cinquièmes le théâtre.

L'œuvre comprend deux actes, répartis en 15 tableaux, et un prologue. Parmi les sujets abordés, la politique canadienne est toujours à l'honneur. Les comptes rendus rapportent un «Duel sur le Champde-mars entre messieurs Tash-Ro et So-vé24 » de même qu'un «Duo du baiser de la paix entre le Chat Félix et la Chatte municipale ».

${ }^{22}$ Les deux hommes collaborent en 1926-1927 à la publication de la revue Le Carillon, bientôt fusionnée à La Lyre. On les retrouve à nouveau en 1930 lors de la présentation du sketch folklorique Une noce canadienne-française en 1830, à l'occasion de la troisième édition, à Québec, du Festival du Canadien Pacifique. Les plans seront toutefois bouleversés par le décès prématuré de Marchand, le $1^{\text {er }}$ mai 1930.

23 La Patrie, 28 février 1925, p. 36; La Presse, 28 février 1925, p. 48; La Patrie, 3 mars 1925, p. 18; La Presse, 3 mars 1925, p. 14; et La Lyre, vol. 3, nº 29 (mars 1925), p. 11. Le centre de conservation de BAnQ, au 2275, rue Holt à Montréal, conserve également un programme officiel de l'œuvre.

${ }^{24}$ Le libéral Louis-Alexandre Taschereau (1867-1952), premier ministre du Québec, et le conservateur Arthur Sauvé (1874-1944), chef de l'opposition officielle à l'Assemblée nationale du Québec. 
L'allusion aux cafés-concerts montmartrois semble sans équivoque en raison du recours à l'allégorie du chat. Un autre sketch de la même revue, "The Gentlemen Farmer de Mol-Hieur [Molière], joué à la manière de... Odéon Gémier [Firmin Gémier, du Théâtre de l'Odéon à Paris] ", fait directement référence à des événements entourant la Soirée chatnoiresque de décembre 1924, dont il était question plus haut ${ }^{25}$.

La distribution de la revue Police... en... quête? permet d'établir d'autres emprunts à la culture du café-concert montmartrois. Roméo Poirier collabore étroitement avec des acteurs clés de la Société canadienne d'opérette (1921-1934), tandis que Jeanne Maubourg (18751953) tient le rôle d'Yvette Guilberto (la diseuse Yvette Guilbert), et que son époux, Albert Roberval (1869-1941), assure la direction de l'orchestre. Camille Bernard, qui participait à la Soirée chatnoiresque, tient, quant à elle, le rôle de la Chatte municipale, et Roméo Poirier intervient lui-même dans le personnage du Chat Félix.

Ceci expliquant cela, on comprend mieux que le répertoire de la revue Police... en... quête? soit composé de timbres sur des airs des compositeurs classiques Charles Gounod (1818-1893), Camille Saint-Saëns (1835-1921), André Messager (1853-1929) et Félix Fourdrain (1880-1923). Il est enfin à noter qu'un certain A. Bourgeois est mentionné comme musicien de l'orchestre de la Société canadienne d'opérette et que Roméo Poirier en est le secrétaire depuis 1922.

\section{En roulant ma boule (1926)}

En janvier 1926, nous retrouvons Albéric Bourgeois et Roméo Poirier au Théâtre Saint-Denis en qualité de coauteurs des textes de la revue d'actualité En roulant ma boule. Cette œuvre porte le même

\footnotetext{
25 Au moment de la Soirée chatnoiresque de décembre 1924, le metteur en scène français Firmin Gémier (1869-1933) et sa troupe du Théâtre National de l'Odéon à Paris sont de passage à Montréal, le temps de quelques soirées de représentation au Théâtre His Majesty's. La troupe donne notamment une représentation du Bourgeois gentilhomme de Molière (voir La Presse, 6 décembre 1924, p. 52; et La Presse, 9 décembre 1924, p. 8).
} 
titre que la rubrique dessinée par Albéric Bourgeois dans les pages de La Presse. On peut en résumer les dix tableaux de la façon suivante : " [L]e père Adam et la mère Ève viennent sur la terre pour étudier le darwinisme et se rendre compte de l'aspect de leurs descendants. Ils en profitent pour passer en revue toutes les actualités $\left[\right.$ de Montréal] ${ }^{26}$. » En plus du choc entre le darwinisme et la Genèse de la Bible, la revue aborde à nouveau la politique canadienne, la prohibition de l'alcool, les journaux et la radio, l'émancipation des femmes, de même que les rapports entre les Canadiens français, les Anglais et les Amérindiens.

La distribution comprend cette fois une cinquantaine de comédiens et de chanteurs, dont Jeanne Maubourg (la Mère Ève), J.-P. Filion (le Père Adam), Élzéar Hamel (Baptiste Ladébauche), Lucille Turner (la Folie), Germaine Giroux (la Princesse Ataka) et un certain Paul Max (le Jeune marié), que nous retrouverons plus loin. La mise en scène est assurée par Raoul Léry (1896-?), revuiste de scène et directeur de la revue Le Canada qui chante. Dans les pages de La Lyre, Henri Letondal affirme qu'En roulant ma boule frise la revue à grand spectacle. Même si les chansons sont amusantes, il subsiste à son avis des longueurs dans l'écriture. Le journal La Presse indiquera quand même que près de 10000 personnes ont applaudi l'œuvre ${ }^{27}$.

\section{0 lions... à l'eau (1927)}

La troisième revue de Roméo Poirier s'intitule Ac...tua...lité/ 14000 lions... [14 millions] à l'eau. Composée de trois actes en douze tableaux, elle est présentée au Théâtre Saint-Denis dans la semaine du $1^{\text {er }}$ mai $1927^{28}$. Au nombre des numéros, "La danse des 1000 lions [millions]... à l'hôtel de ville " dissèque un scandale politique et financier à Montréal. Le tableau l'" Apothéose de la confédération " permet par ailleurs de souligner les soixante ans de fondation du

${ }^{26}$ La Patrie, 28 janvier 1926, p. 18.

27 La Lyre, vol. 4, no 37 (février 1926), p. 5; et La Presse, 21 avril 1927, p. 20.

28 La Presse, 21 avril 1927, p. 20; et La Lyre, vol. 5, n 50 (mars 1927), p. 28. 
Canada moderne. La mise en scène est de nouveau assurée par Raoul Léry. L'orchestre revient sous la baguette d'Albert Roberval tandis que le chœur est dirigé par Céline Marier (v. 1871-1940). La musique, arrangée conjointement par maints auteurs, reprend des extraits d'opéras et des airs populaires. La critique se réjouit de l'énorme succès d'assistance dans la salle du Théâtre Saint-Denis ${ }^{29}$.

Tentons une synthèse des collaborations artistiques à l'œuvre ci-dessus. Les auteurs de sketches et de chansons d'actualité Roméo Poirier et Albéric Bourgeois sont bien épaulés par la musique des membres en règle de la Société canadienne d'opérette, à commencer par les époux Jeanne Maubourg et Albert Roberval. Leurs principaux collaborateurs habituels sont des chanteurs de formation classique et des acteurs, parfois les deux : Camille Bernard, Charles Marchand, Germaine Giroux, Raoul Léry et ainsi de suite. D'autres artistes qui interviennent lors de l'une ou l'autre de ces revues d'actualité vont devenir des têtes d'affiche du cabaret Au matou botté ${ }^{30}$. La culture des cafés-concerts montmartrois se manifeste par le traitement satirique de la chose politique, le travail sur des timbres d'opérette ou d'airs connus et, bien évidemment, par ce motif allégorique du " chat », qui demeure omniprésent. Tant dans le choix du répertoire que dans celui du personnel, la majorité des personnes nommées vont jouer un rôle actif dans la première mouture du cabaret $\mathrm{Au}$ matou botté.

\section{De Montmartre à Montréal : un nouveau café-concert pour la chanson française}

L'ouverture du cabaret Au matou botté a lieu le 26 novembre 1929 dans la Salle des Châteaux du restaurant français Kerhulu \& Odiau,

29 La Presse, 21 avril 1927, p. 20; La Presse, 30 avril 1927, p. 45; et La Presse, 3 mai 1927 , p. 17.

${ }^{30}$ Louis Chartier, Charles-Émile Brodeur, Fred Barry, Germaine Daignault, Georges Legrand et Louis-Philippe Hébert. 


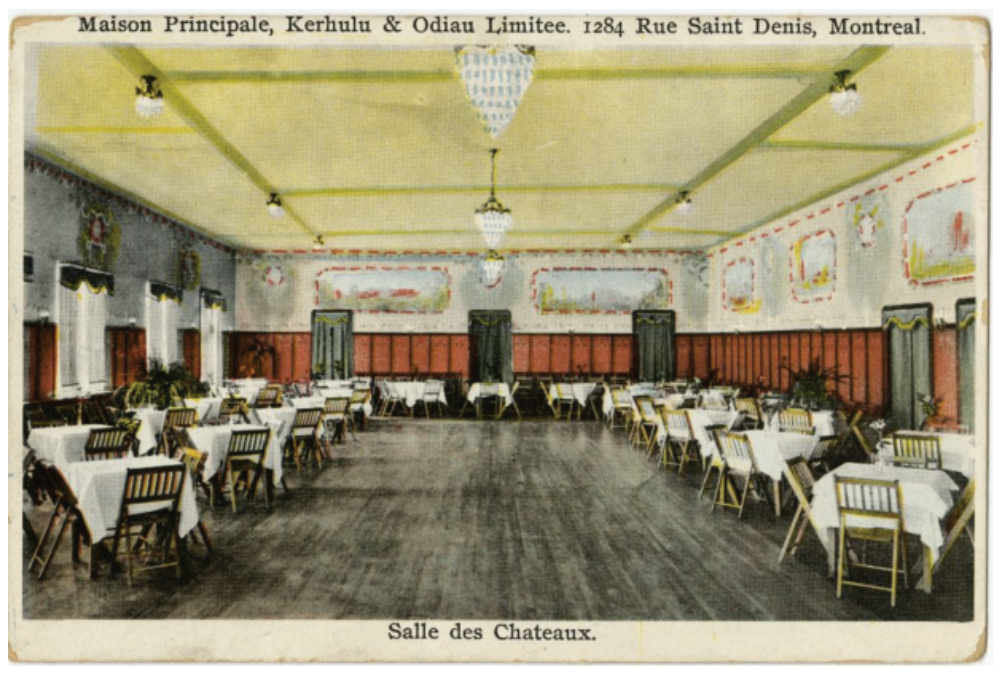

Figure 1 - « Maison principale, Kerhulu \& Odiau limitée, 1284 rue SaintDenis, Montréal : Salle des Châteaux » ([Anonyme], carte postale, [192-?], Collection numérique de BAnQ, CP 029949 CON, [En ligne], [http://www. banq.qc.ca/collections/images/notice.html?id=0004387894]). Le cabaret Au matou botté y loge entre 1929 et 1932.

situé au 1284, rue Saint-Denis, dans le Quartier latin de Montréal (voir la figure 1$)^{31}$.

Dans les annonces publicitaires, la nouvelle «boîte montmartroise » de la métropole utilise l'icône d'un chat noir, placé debout

31 Joseph Kerhulu (1875-1957) et Alphonse Odiau sont les fondateurs et propriétaires des différents restaurants Kerhulu \& Odiau, à Montréal et à Québec. Dans sa thèse de doctorat sur l'histoire de la danse récréative à Montréal, Peggy Roquigny explique que le premier établissement ouvre ses portes sur Saint-Denis en 19111912. Toutes les succursales offrent le restaurant, la pâtisserie, le salon de thé et le service de banquet. En 1926, on convie la clientèle à un "grand souper danse [avec] orchestre et autres amusements ". Dès lors, Kerhulu \& Odiau intègre de façon permanente la danse à la restauration, le cabaret en soirée et les thés dansants en après-midi (Roquigny, Les plaisirs de la danse à Montréal, p. 285-287; voir également "Les amis de la Revue moderne ", La Revue moderne, vol. 4, n 12 (octobre 1923), p. 14-15; ainsi que Jean-Marie Lebel, " Il y a 50 ans... le Vieux-Québec ", Cap-aux-Diamants : la revue d'histoire du Québec, numéro hors série (automne 1999), p. 10-15). 


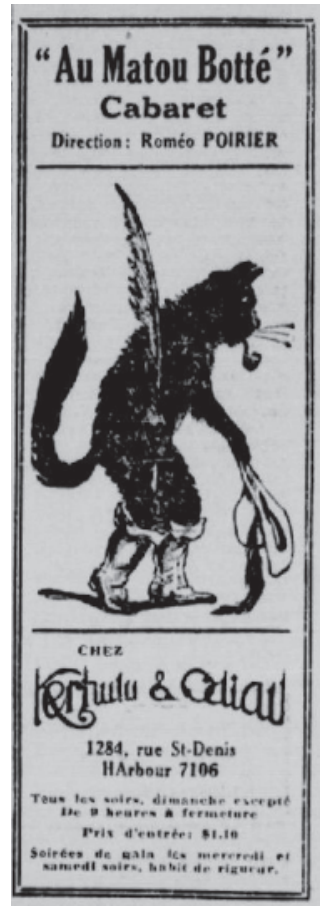

Figure 2 - Publicité du cabaret Au matou botté, La Patrie, 30 novembre 1929 , p. 36. et de profil (voir la figure 2). L'animal au dos rond porte des bottes, une pipe en bouche, une grande plume à la main droite et un chapeau à la gauche. Le dessin est vraisemblablement une création d'Albéric Bourgeois. En raison du nom donné au cabaret, on pense immédiatement au célèbre conte du Chat botté dans la version de Charles Perrault (1628-1703). Et en observant plus attentivement la silhouette du chat, on constate qu'elle n'est pas sans rappeler le dessin du Vieux de 37 d'Henri Julien (1852-1908) ${ }^{32}$. Ces deux figures ont au moins en commun la posture de profil, la pipe et le port d'arme un fusil et une plume de caricaturiste. Imaginons que la grande plume est celle d'Albéric Bourgeois; le matou botté se pose alors tel un avatar de Baptiste Ladébauche, son coloré personnage des chroniques de La Presse. Autrement dit, le cabaret français aura une couleur canadienne assumée.

Le nouveau cabaret Au matou botté est placé sous la direction de Roméo Poirier (voir la figure 3), avec la collaboration de Bourgeois.

Voici ce que rapporte le journal La Patrie au sujet de la soirée inaugurale :

Hier soir, chez Kerhulu, nos excellents camarades Roméo Poirier et Albéric Bourgeois ont inauguré leur cabaret chantant, "Au Matou Botté ", qui a remporté le plus franc succès. On a refusé du monde, ce qui est certainement très bon signe. [...] Il était précédé de deux formules, celle de M. André Dahl : « Gaîté, bonne

\footnotetext{
32 Mes remerciements à l'historien de l'art Dominic Hardy de l'Université du Québec à Montréal pour cette observation judicieuse.
} 


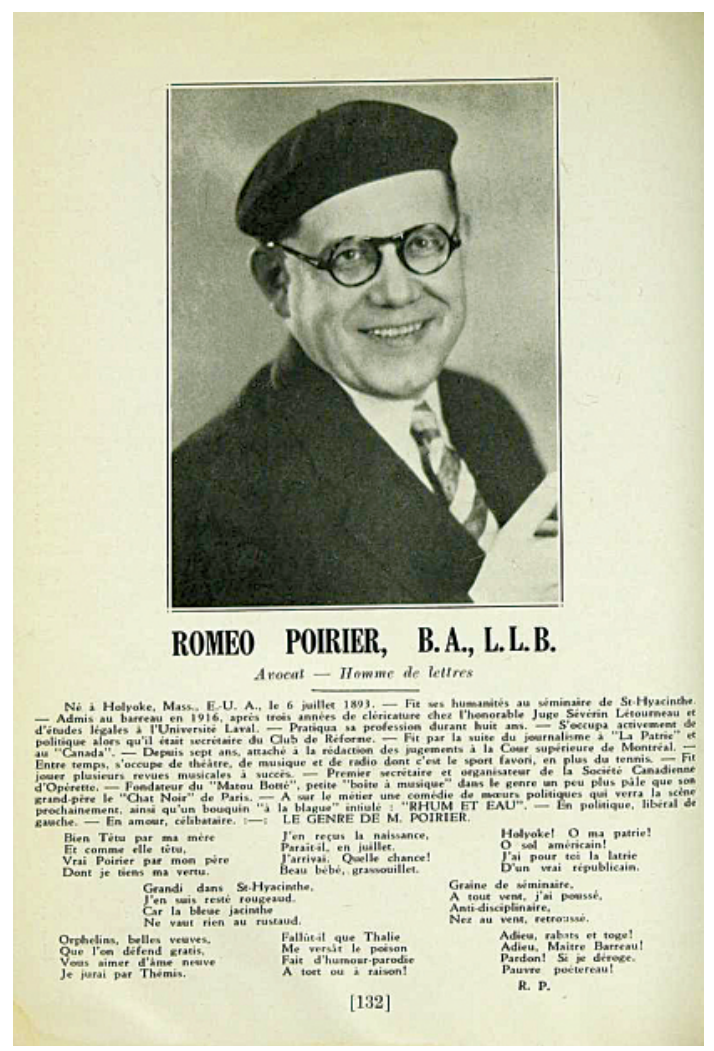

Figure 3 - " Roméo Poirier ", dans Raphaël Ouimet, Biographies canadiennes-françaises, $11^{\mathrm{e}}$ édition, Montréal, [s. é.], 1933, p. 132 (Collection numérique, BAnQ, [En ligne], [http://collections.banq.qc.ca/ ark:/52327/2634224]).

gaîté, toi sans qui la vie n'est qu'un sommeil, tu te fais rare! » et celle de M. Édouard Montpetit : « Le Restaurant-boîte, orné de mille choses agréables, que ne le tentons-nous ici " ${ }^{33}$ !

Dans La Presse, Joseph Kerhulu est l'objet d'un long article avec une grande photographie, où il se proclame " ambassadeur de la com-

33 La Patrie, 26 novembre 1929, p. 7. 
mune libre de Montmartre ${ }^{34} \gg$. Quelques jours plus tard, le Montreal Daily Star souligne lui aussi l'inauguration du « New French Cabaret » :

For the encouragement of cheerfulness in Montreal a new cabaret has been opened - a cabaret in the French style, rather different from other Montreal cabarets. Under the name of "Le Matou botte " [sic] it is held in the large room above Kerhulu and Odiau's on St. Denis Street, and the room has been amusingly decorated with all sorts and kinds of black cats, caricatures of Montreal celebrities and other attractions, with more still to come $e^{35}$.

Pendant les cinq premiers mois, on réunit Au matou botté une troupe régulière d'environ quinze chanteurs, comédiens et danseuses, la plupart locaux, tous dirigés par Bourgeois et Poirier. Chez les chanteurs - paroliers et musiciens inclus -, on trouve Gilles-René Amiot $^{36}$, Lucille Turner, Jean Claver et Ernest Loiselle, le maître de cérémonie des lieux. Chez les acteurs - auteurs de monologues et de sketches comiques -, il faut compter Jules Ferland, Fred Barry, Madeleine Davies, Tony Labelle, M. Dauriac ${ }^{37}$ et Paul Max, dont nous avons croisé le nom plus haut. Enfin, les danseuses et ballerines vedettes sont les sœurs d'Arcy ${ }^{38}$ et les Rosebuds ${ }^{39}$.

${ }^{34}$ La Presse, 30 novembre 1929, p. 54.

35 Montreal Daily Star, 3 décembre 1929, p. 8.

36 À propos de Gilles-René Amiot, voir Biographies canadiennes-françaises, $1^{\text {re }}$ éd., Ottawa, J. A. Fortier, 1920, p. 406.

37 Le prénom de cet acteur n'est jamais donné dans les sources.

38 Les sœurs d'Arcy, ou D'Arcy Sisters (Germaine Daignault et Antonia " Tony " La France) sont deux élèves de Maurice Lacasse-Morenoff (voir La Patrie, 29 septembre 1930 , p. 7).

39 Les Rosebuds, ou Canadian Rosebuds, sont quatre jeunes danseuses de genre dans les cabarets montréalais de l'époque. Leur groupe se compose des deux sœurs Merrill et des deux sœurs Campbell. Je n’ai pas trouvé de détail sur les deux premières. Quant à Eleanor et Bernice "Bee " Campbell, ce sont des jumelles écossaises employées au Théâtre National, aux côtés de la danseuse Effie Mack, femme d'Olivier Guimond père. Elles travaillent également au Venetian Gardens. En entrevue à La Patrie en février 1930, elles révèlent leur ambition : "Qu’idéalisez-vous au juste? Où visez-vous? Demandons-nous. "Le Broadway!", admet Bernice souriante. — "Le Broadway!” souligne sérieusement Eleanor » ( $\mathrm{La}$ Patrie, 15 février 1930, p. 23; et L'œil en coulisse, no 2 (29 mars 1930), p. 12). 
Durant la semaine qui suit l'ouverture, le programme comprend des chansons comiques interprétées par Roméo Poirier, Gilles-René Amiot et Lucille Turner ${ }^{40}$, une pochade d'Albéric Bourgeois ${ }^{41}$, la revue Bottons le matou par Madeleine Davies, des sketches de Jules Ferland, Fred Barry et Paul Max, de même que des danses de fantaisie exécutées par les sœurs d'Arcy. Le prix d'entrée est fixé à 1,10\$. Le cabaret est ouvert tous les soirs à compter de 21 heures, excepté le dimanche.

À la troupe régulière s'ajoutent chaque semaine un ou deux invités spéciaux, à l'occasion des soirées de gala qui ont lieu les mercredis et samedis soirs ${ }^{42}$. Ces soirées sont précédées par un thé dansant qui se déroule de 16 à 19 heures ${ }^{43}$. L'équilibre entre le programme habituel et les invités spéciaux semble acquis dès le départ. Durant sa première semaine d'existence (26-30 novembre 1929), Au matou botté accueille Madame Aimée Morin, une diseuse et chanteuse de la boîte La Vache, à Paris ${ }^{44}$. Au cours de la deuxième semaine (2-7 décembre), Aimée Morin partage la vedette avec Gaston Saint-Jacques, un membre de la Société canadienne d'opérette qui propose des imitations de Maurice Chevalier (1888-1972) et une truculente fantaisie intitulée "Noces journalistiques ${ }^{45}$ ".

À la troisième semaine (9-14 décembre), le cabaret s'adjoint les services d'Eugène Vivez, un diseur, chanteur, comédien et danseur de l'Olympia de Paris ${ }^{46}$. Ce personnage, on le verra, jouera un rôle majeur dans l'évolution du cabaret Au matou botté. En plus de la pochade chatnoiresque, Roméo Poirier présente le sketch «La bourse ou l'avis ", une fantaisie librement adaptée de la fable La cigale et la fourmi de Jean de La Fontaine (1621-1695). Albéric Bourgeois organise, pour sa part, un "Concours de beauté masculine ». Alors

\footnotetext{
40 Avec la reprise d'une chanson de Lucienne Boyer.

${ }^{41}$ Vraisemblablement inspirée de la Soirée chatnoiresque de décembre 1924.

${ }^{42}$ La Patrie, 29 novembre 1929, p. 16; et La Patrie, 30 novembre 1929, p. 36.

43 La Patrie, 4 décembre 1929, p. 7.

44 La Presse, 30 novembre 1929, p. 75.

45 La Presse, 3 décembre 1929, p. 8 ; et La Patrie, 3 décembre 1929, p. 4.

46 La Presse, 7 décembre 1929, p. 69; et La Patrie, 11 décembre 1929, p. 4.
} 


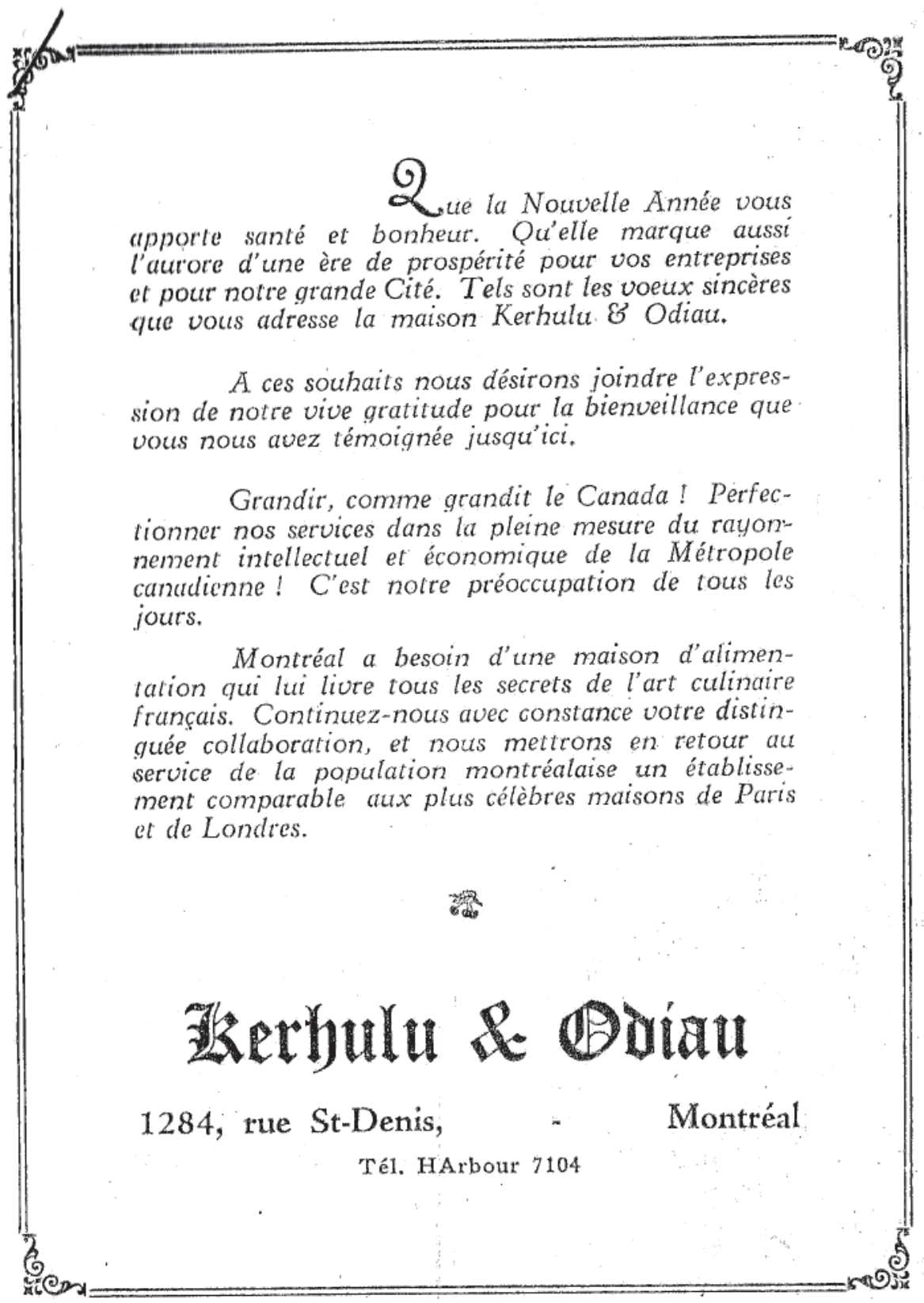



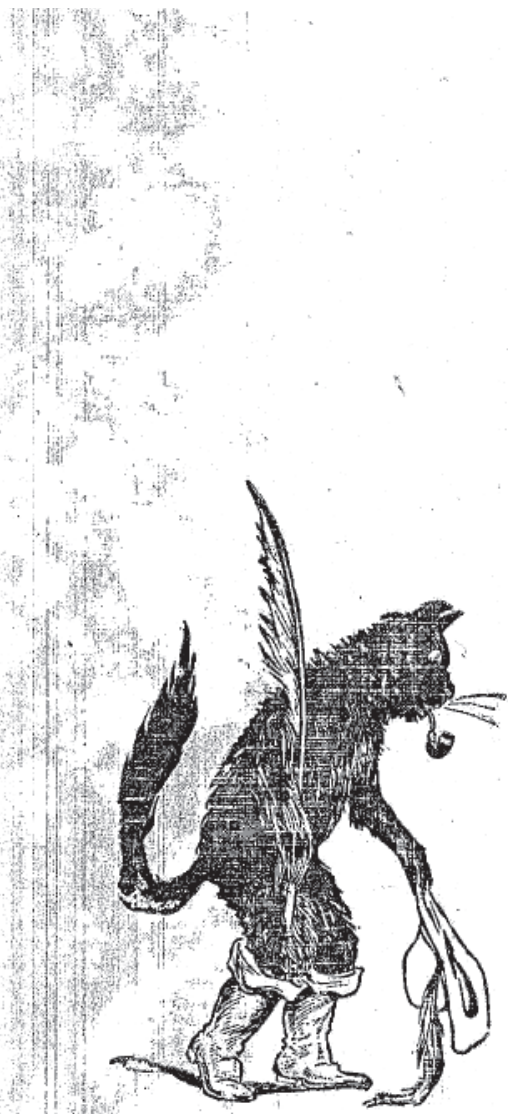

Figure 4 - Extrait d'un programme du jour de l'An du cabaret Au matou botté, chez Kerhulu \& Odiau (BAnQ VieuxMontréal, Fonds Albéric-Bourgeois, MSS346, 2006-10-001, 2564). Les paroles de la chanson thème "Mont' chez nous " sont également reproduites dans ce programme. 
que Jules Ferland présente le sketch "Les matous de la $11^{\mathrm{e}}$ heure ", Lucille Turner monte sur scène déguisée en Matouse. On le voit, les chansons de Paris, la satire et le thème du chat demeurent des repères constants dans la programmation du café-concert.

Au cours de la quatrième semaine (16-21 décembre), Roméo Poirier crée le sketch « Chez Rubin, 1939 rue Craig Ouest », librement adapté d'une pièce d'Henri Letondal intitulée Chérubin $1930^{47}$. À la soirée de gala du samedi, Charles Marchand est l'artiste invitét ${ }^{48}$. On adopte aussi un refrain officiel du cabaret Au matou botté, sur l'air « Tu verras Montmartre (Monte là-dessus) » de Lucien Boyer (1876-1942). Les paroles en sont reproduites dans un programme (voir la figure 4) :

"Mont' chez nous », le refrain de la boîte Au matou botté

Mont' chez nous, Mont' chez nous, Mont' chez nous,

Tu verras Montmartre

Viens chez nous, viens chez nous

Tu verras sûr'ment que'que chose de fou

Du vrai Matou

Et sois bien convaincu

Qu'on n'y dira pas d'histoires de...

Soyez bienvenus en gala ou tous nus,

Au matou botté... té... tét ${ }^{\prime 9}$.

47 Dans mon article « Du théâtre en chanson? La transfolklorisation chez les collaborateurs du Carillon canadien de Charles Marchand "(Les Cahiers de la société québécoise de recherche en musique, vol. 14, $\mathrm{n}^{\circ} 2$ (automne 2013), p. 45, note 31), j'avais erronément attribué le sketch "Chez Rubin, 1939 rue Craig Ouest » à Henri Letondal. L'adaptation de la pièce de Letondal, signée par Roméo Poirier, est confirmée dans La Presse, 14 décembre 1929, p. 63.

48 La Patrie, 16 décembre 1929, p. 8. Rappelons que Marchand était complice de la Soirée chatnoiresque de décembre 1924.

49 Pour un enregistrement sur disque 78 tours de Tu verras Montmartre (Monte là-dessus), réalisé en 1925 par Lucien Boyer, consulter YouTube : [https://www. youtube.com/watch?v=lrtHMMb863s]. 


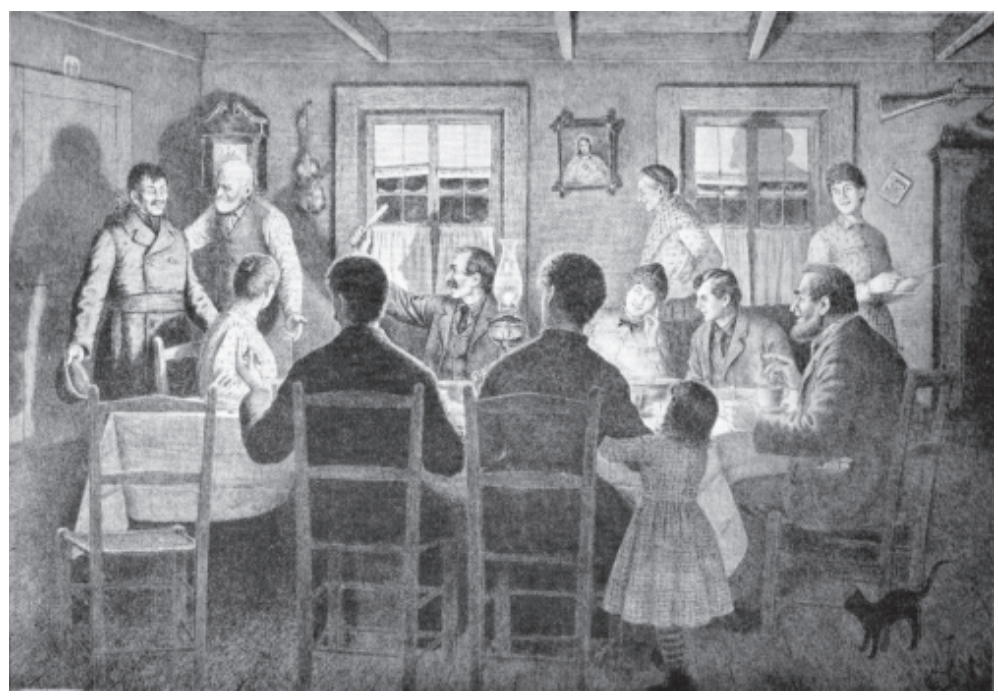

Figure 5 - Edmond-Joseph Massicotte, « Le Réveillon de Noël », dans Nos Canadiens d'autrefois: 12 grandes compositions, Montréal, Granger Frères, 1923, p. 13. On remarque immédiatement le chat noir au dos rond, placé en bas, à droite.

Le cabaret Au matou botté organise ensuite un grand gala de Noël pour le 24 décembre 1929. À cette occasion, le comédien Fred Barry (1887-1964) dirige un tableau vivant à propos de la messe de minuit, inspiré d'une œuvre du peintre Octave Bélanger (1886-1972) - ce pourrait être la peinture $\grave{A}$ la sortie de la messe. Un autre numéro est basé sur le tableau Le Réveillon de Noël de l'illustrateur EdmondJoseph Massicotte (1875-1929), bien connu pour ses représentations des Canadiens d'autrefois (voir la figure 5) ${ }^{50}$. La diseuse Aimée Morin, le baryton Louis Chartier et le "sifflomane " René Poirier accompagnent la troupe régulière lors de cette soirée.

Pour la Veillée du jour de l'An du 31 décembre 1929, on concocte un autre réveillon sur scène. Bourgeois et Poirier dirigent cette fois une reconstitution du tableau La bénédiction du jour de l'An du même

50 La Patrie, 24 décembre 1929, p. 4. 
Edmond-Joseph Massicotte ${ }^{51}$. À cela s'ajoute un tableau sur la fin de 1929, un numéro de "notre si gentille "matouse" ", Lucille Turner, le "siffleur émérite " René Poirier et une apparition de la comédienne Germaine Giroux, établie à New York ${ }^{52}$. Bien sûr, toute la troupe régulière est toujours de la partie.

Au début de janvier 1930, la programmation inclut les sketches "Les matous artistiques » et " La bataille du siècle entre Idola SaintJean et le Code civil de la province de Québec ${ }^{53}$ ». Nous avons vu plus haut que la satire politique est fréquemment à l'honneur au café-concert. Ici, le thème du chat est jumelé à un sketch comique sur le droit de vote des femmes. La semaine suivante, on présente une " Parodie de l'annonceur de radio au poste K.C.K.C. [CKAC] ", dans laquelle Roméo Poirier doit imiter le commentateur de hockey ${ }^{54}$. On annonce aussi la venue prochaine à Montréal de Maurice Chevalier, sous la direction de Roméo Poirier. Ce dernier étant occupé avec la vedette française, on comprend mieux que le cabaret Au matou botté ne donne que deux soirées cette semaine-là (voir la figure 6). La culture du café-concert montmartrois continue de se faire valoir avec sa couleur canadienne dans les sketches, les personnages et les décors.

Bourgeois et Poirier n'hésitent pas à créer des événements spéciaux ou à promouvoir l'image d'un lieu bien fréquenté par le ToutMontréal. Au début de février 1930, on organise un premier grand «Bal-matou », une sorte de soirée costumée ${ }^{55}$. Un mois plus tard, un «Grand bal hystérique » pour les jours gras s'annonce ${ }^{56}$. Et l'on

51 La Patrie, 28 décembre 1929, p. 40; et Edmond-Joseph Massicotte, «La bénédiction du Jour de l'An ", Nos Canadiens d'autrefois : 12 grandes compositions, Montréal, Granger Frères, 1923, p. 10.

52 La Patrie, 26 décembre 1929, p. 4; et La Patrie, 28 décembre 1929, p. 40.

53 La Presse, 4 janvier 1930, p. 59; et Montreal Daily Star, 4 janvier 1930, p. 20.

${ }^{54}$ Ce sketch avait déjà été présenté en mars 1925, dans la revue Police... en... quête? de Poirier (voir La Presse, 11 janvier 1930, p. 65; et La Patrie, 11 janvier 1930, p. 36).

55 La Patrie, 12 février 1930, p. 8.

56 La Patrie, 27 février 1930, p. 8; et La Patrie, 4 mars 1930, p. 8. 


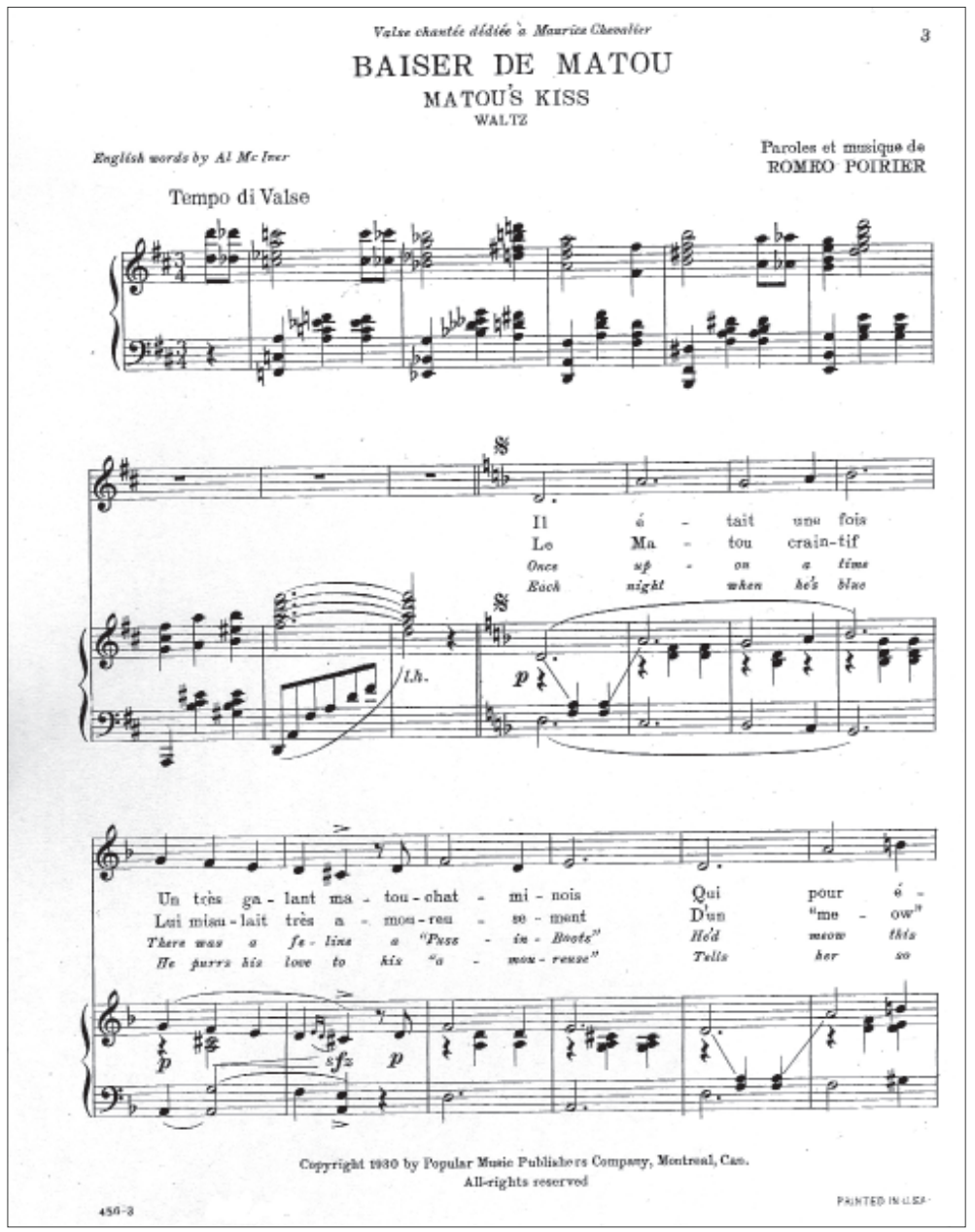

Figure 6 - Roméo Poirier, paroles et musique. Première page de la feuille de musique de la chanson Baiser de matou/Matou's Kiss, Montréal, Popular Music Publishers, 1930. Valse chantée, dédiée à Maurice Chevalier. Paroles anglaises d'Allan McIver, pianiste du Trio lyrique de Lionel Daunais. Partition conservée à la Collection nationale de musique, BAnQ.

renchérit à la Mi-Carême avec un "Bal Qu'os-tu mets? " (Bal costumé), au son de l'orchestre de Léo Le Sieur (1897-1983) ${ }^{57}$. Une soirée

57 La Patrie, 8 mars 1930, p. 20 ; et La Patrie, 22 mars 1930, p. 22. 
spéciale permet aussi, le 17 mars, de souligner la Saint-Patrice (SaintPatrick), fête nationale des Irlandais ${ }^{58}$.

Dès l'ouverture, Albéric Bourgeois avait repris sa pochade chatnoiresque de 1924 tout en proposant de réaliser des dessins de maires et autres notables en direct ${ }^{59}$. Les communiqués de presse font d'ailleurs la promotion de la boîte en valorisant l'image française et en énumérant les noms des personnalités de la société montréalaise qui sont présentes ${ }^{60}$ : par exemple, l'honorable juge Édouard FabreSurveyer (1875-1957); maître Guillaume Saint-Pierre et ses « quinze amis »; et Pierre Beaubien, qui dirige à la radio l'émission folklorique de la Brasserie Frontenac ${ }^{61}$. Même le maire Camilien Houde (18891958 ) et le Consul de France viennent y faire une visite ${ }^{62}$. L'enthousiasme de la critique pour le répertoire à l'affiche est bon pour les affaires :

Mais remarque-t-on assez avec quel goût les chansons, les danses et les sketches sont présentés? On n'a qu'à faire une comparaison entre le « Matou botté » et les autres établissements du genre pour conclure que l'esprit français, sa simplicité et sa clarté sont parfaitement reflétés dans les décorations, les costumes et le caractère des numéros donnés ${ }^{63}$.

Jusqu'en mai 1930, l'image médiatisée du cabaret Au matou botté de Bourgeois et Poirier demeure donc celle d'un lieu unique à Montréal pour ceux qui veulent se divertir comme à Montmartre. Les chansons sur des airs d'opérette française, les sketches d'actualité et les thés dansants incarnent très bien cet esprit gaulois. Mais avec la crise économique qui sévit, peut-il être longtemps rentable de résister au divertissement Made in America?

\footnotetext{
58 La Patrie, 18 mars 1930, p. 6.

59 La Presse, 29 novembre 1929, p. 12.

60 La Patrie, 12 décembre 1929, p. 16.

${ }^{61}$ Luc Bellemare, "Notre folklore "au radio": $1^{\text {re }}$ partie : l'émission de la Brasserie Frontenac à CKAC ", Bulletin Mnémo, vol. 13, no 4 (automne 2012), p. 1-5.

${ }^{62}$ La Patrie, 13 janvier 1930, p. 8 ; et La Presse, 15 janvier 1930, p. 8.

${ }^{63}$ La Presse, 31 décembre 1929, p. 8.
} 


\section{Vers un virage esthétique et une relève au cabaret?}

Lorsque La Patrie indique, en janvier 1930, que l'orchestre de la boîte Au matou botté « joue du jazz, soit, mais pas de ce jazz tonitruant qui vous fait craindre pour vos tympans ", on ne fournit guère de détails qui permettent d'approfondir la portée de l'opinion exprimée $^{64}$. En recoupant ce passage avec d'autres textes critiques de l'époque, on peut toutefois y lire entre les lignes que l'auteur affiche une préférence pour le jazz symphonique avec grand orchestre, celui prisé à New York par Paul Whiteman (1890-1967) ou par George Gershwin (1898-1937), par exemple. Comme en musique classique, ce jazz favorise la mélodie et le jeu de timbres des familles d'instruments - bois, vents, cuivres, cordes. Il s'oppose ainsi aux petits orchestres de danse et au répertoire afro-américain d'Harlem, dont le rythme effréné va bientôt gagner toute l'Amérique du Nord. Globalement, la critique montréalaise préfère encore la musique classique et l'opéra.

En effet, on inaugure pendant la saison hivernale 1930 une toute nouvelle série de récitals lyriques, présentés les dimanches après-midis Au matou botté. La soprano Camille Bernard, les barytons Louis Chartier et Georges Bétournay, de même que la soprano Germaine Bruyère y proposent un répertoire de mélodies françaises et d'airs harmonisés par le folkloriste français Jean-Baptiste Weckerlin (1821$1910)^{65}$.

On peut, à raison, se demander si, une fois passé l'attrait de la nouveauté, l'effervescence de la jeune boîte montréalaise ne commence pas à faiblir. Depuis l'ouverture, la direction artistique n'a pourtant pas ménagé ses efforts afin de présenter d'anciens collaborateurs comme des attractions exceptionnelles. Il faut également mentionner

64 La Patrie, 14 janvier 1930, p. 4.

65 La Patrie, 4 janvier 1930, p. 36; La Patrie, 20 janvier 1930, p. 7; La Patrie, 29 janvier 1930, p. 4 ; Montreal Daily Star, 17 janvier 1930, p. 23 ; et La Lyre, vol. 7 , $\mathrm{n}^{\circ} 70$ (décembre 1929-janvier 1930), p. 16 et 21. 
la présentation de certains anciens sketches recyclés par Bourgeois et Poirier $^{66}$.

Les signes d'effritement de la troupe fondatrice sont pourtant assez nombreux, si l'on s'en tient aux traces visibles dans les journaux. Le nom d'Albéric Bourgeois ne paraît plus dans les communiqués de presse après le 2 janvier 1930, mais on sait qu'il s'engage alors avec Charles Marchand dans l'écriture d'Une noce canadienne-française en 1830 (revoir note 22). Celui du chansonnier Gilles-René Amiot ne sera plus mentionné après le 18 février 1930. Ceux de Jules Ferland et de Roméo Poirier n'ont pas reparu après le 29 avril 1930 ${ }^{67}$. Et le nom de Lucille Turner disparaît des communiqués du cabaret Au matou botté après le 22 juillet $1930^{68}$.

\section{La parenthèse du magazine L'œil en coulisse (1930)}

Le cabaret est plus calme pendant les six semaines du Carême, dit-on, mais on annonce néanmoins une grande "Fête montmartroise " pour le début d'avril $1930^{69}$. Alors que les quotidiens La Patrie et La Presse ne relaient presque plus d'informations concernant le Matou botté, on peut se tourner vers l'éphémère périodique L'œil en coulisse ${ }^{70}$. Ce magazine de 32 pages est essentiellement composé d'entrevues avec des artistes, d'anecdotes de scène, de plaisanteries et de critiques, le tout " entièrement rédigé par un seul homme... comme "Le

66 La Patrie, 2 mai 1930, p. 8.

${ }^{67}$ Voir, par exemple, La Patrie, 6 septembre 1930, p. 21. Il subsiste dans les publicités des journaux quelques mentions de Poirier à la "direction " du cabaret. Ces indications, on le verra plus en détails dans les pages suivantes, contredisent le contenu des communiqués de presse. En revanche, l'association perpétuée au nom du fondateur ne nuisait probablement pas à l'image de l'entreprise, bien au contraire.

68 Dans La Patrie, 22 octobre 1930, p. 8, on annonce que Lucille Turner est passée à la Société canadienne d'opérette.

69 La Patrie, 9 avril 1930, p. 8; et La Patrie, 29 avril 1930, p. 8.

70 L'oeil en coulisse, $\mathrm{n}^{\circ}$ 1, 22 mars 1930; n², 29 mars 1930; et no 3, 5 avril 1930. La revue est conservée à la section des périodiques de la Collection nationale de $\mathrm{BAnQ}$, à Montréal. 
Goglu"71 ». Il apparaît assez plausible qu'Henri Letondal soit le maître d'œuvre de cette initiative.

Sans le dire explicitement, L'œil en coulisse fait figure d'organe de promotion du cabaret Au matou botté. La revue a pignon sur rue au 1284 , rue Saint-Denis ${ }^{72}$, soit l'adresse du restaurant Kerhulu \& Odiau et de la boîte montmartroise. Les contenus du magazine portent sur la plupart des membres de la troupe régulière et des proches collaborateurs : le directeur Roméo Poirier, les chanteurs, les auteurs de sketches et les danseuses, sans oublier l'orchestre de Léo Le Sieur. Eugène Vivez et Paul Max, les deux hommes qui vont bientôt prendre la direction artistique du cabaret, sont également à l'honneur.

Des textes sur la radiophonie publiés dans L'œil en coulisse, judicieusement signés du pseudonyme T. Esseffe (TSF) ${ }^{73}$, révèlent l'existence d'une certaine complicité avec l'émission L'heure provinciale $^{74}$, la seule du poste CKAC qui soit financée par des fonds publics pendant l'entre-deux-guerres. De 1929 à 1939, la programmation de L'heure provinciale, dirigée par Letondal, privilégie la musique classique et le chant lyrique, mais la chanson française, par l'intermédiaire du segment Les refrains que l'on aime entendre, y occupe aussi une place. En janvier 1937, le même Letondal présentera la revue de café-concert Mon Paris à la Salle des banquets du restaurant Kerhulu \& Odiau ${ }^{75}$.

${ }^{71}$ La revue Le Goglu est l'un des hebdomadaires de propagande nazie publiés au Québec dans les années 1930 par Adrien Arcand (1899-1967). Voir L'oil en coulisse, $\mathrm{n}^{\circ} 1,22$ mars 1930 , p. 3.

72 L'oil en coulisse, $\mathrm{n}^{\circ} 1,22$ mars 1930, p. 32.

73 La TSF, ou télégraphie sans fil, est l'ancêtre de la radiodiffusion. À l'époque, le sigle est courant.

74 L'oeil en coulisse, ${ }^{\text {os }} 1$ à 3, p. 21 ; et Luc Bellemare, "Notre folklore "au radio": $3^{e}$ partie: l'émission L'heure provinciale à CKAC », Bulletin Mnémo, vol. 14, n 2 (hiver 2014), p. 7-13.

75 Voir Sicotte, Gratien Gélinas, p. 103; et La Presse, 16 janvier 1937, p. 37. 
D’après le premier numéro de L'oil en coulisse, Roméo Poirier serait sur le point de fonder un second cabaret ${ }^{76}$, mais aucune autre source ne permet de le confirmer. On présente ensuite une entrevue avec Irène Trudeau ${ }^{77}$, une élève de Louis Chartier et cousine germaine de la grande cantatrice canadienne-française Emma Albani (18471930), qui va mourir le 3 avril.

En page titre du deuxième numéro paraît une caricature du comédien Fred Barry, un dessin en apparence de la plume d'Henri Letondal. Dans une entrevue avec Eugène Vivez, on revient brièvement sur le scandale suscité par les paroles embarrassantes du refrain de la chanson Valentine, chantée par Maurice Chevalier lors de sa visite à Montréal. Afin d'éviter de heurter les oreilles sensibles, le diseur français affirme avoir subtilement changé le mot original " tétons " en " petons ${ }^{78}$ ". Au troisième et dernier numéro, on trouve un éditorial contre l'accordéon, cette " nouvelle épidémie, bien plus cruelle encore que celle des batteurs de semelles [les danseurs de gigue et de claquette] dans les music-halls montréalais ${ }^{79}$ ".

Toute cette première partie de l'histoire du cabaret $\mathrm{Au}$ matou botté donne une vision qui s'accorde relativement bien avec ce que rapporte le court article de Gilles Potvin sur Henri Letondal dans l'Encyclopédie de la musique au Canada ${ }^{80}$. À Paris comme à Montréal, le café-concert à la mode de Montmartre valorise en somme la chanson française et les diseuses, l'opérette, les sketches et la satire politique, les thés dansants et le thème du « chat".

${ }^{76}$ L'eil en coulisse, $\mathrm{n}^{\circ}$ 1, 22 mars 1930, p. 3.

77 Ibid., p. 5.

78 L'oil en coulisse, n' 2, 29 mars 1930, p. 2.

${ }^{79}$ L'oeil en coulisse, $\mathrm{n}^{\circ} 3,5$ avril 1930, p. 23.

80 Sauf pour la fondation du café-concert, que Potvin attribue erronément à Henri Letondal, et la période d'activités du lieu, qu'il limite à l'année 19301931 (voir Gilles Potvin, "Letondal, Henri ", L’Encyclopédie de la musique au Canada, dernière modification : 16 décembre 2013, [En ligne], [http://www. thecanadianencyclopedia.ca/fr/article/henri-letondal-emc/]). 


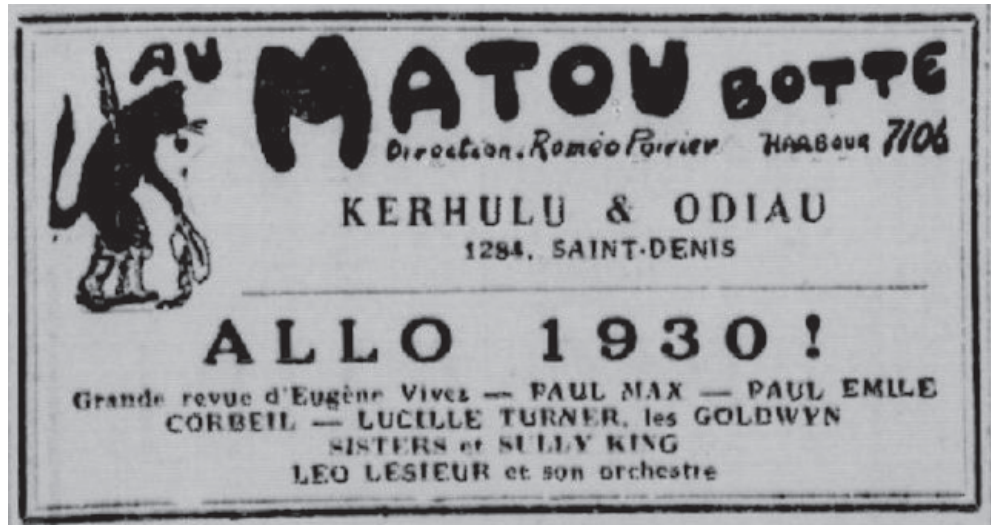

Figure 7 - Annonce publicitaire parue dans La Patrie, 14 juin 1930, p. 22.

\section{De Broadway à Montréal : place au petit orchestre de jazz pour la danse}

Au printemps 1930, Eugène Vivez et Paul Max vont apporter au Matou botté une toute nouvelle esthétique, celle d'un nightclub de Broadway (voir la figure 7).

Le journal La Patrie rapporte, en février 1930, que Roméo Poirier et les artistes de son cabaret artistique sont allés à New York pour participer aux fêtes de la Société Saint-Jean-Baptiste ${ }^{81}$. Un mois plus tard, la Société Saint-Jean-Baptiste de New York a de nouveau requis les services de Roméo Poirier, Lucille Turner et Ernest Loiselle ${ }^{82}$. Cette collaboration entre Montréal et New York laisse forcément un vide dans la programmation normale du café-concert.

$\mathrm{Si}$, dans les publicités parues dans les journaux, Roméo Poirier demeure, pendant un certain temps, le directeur du cabaret Au matou botté, la troupe régulière de Montréal a singulièrement changé de visage $^{83}$. Ce sont maintenant Paul Max et Eugène Vivez qui assurent

${ }^{81}$ La Patrie, 12 février 1930, p. 8.

82 La Patrie, 8 mars 1930, p. 20.

${ }^{83}$ Voir, par exemple, La Patrie, 6 septembre 1930, p. 21. Sous le nom de Roméo Poirier, la publicité clame : "Le cabaret le plus populaire de Montréal. Mieux et plus fréquenté que jamais. " (En italique dans le texte.) 
la direction artistique de l'établissement ${ }^{84}$. Collaborent avec eux les chanteurs Paul-Émile Corbeil et Lucille Turner, de même que les danseuses Marguerite Bray, les Goldwin Sisters et Sally Kane ${ }^{85}$.

La transformation du cabaret Au matou botté en nightclub de Broadway se fait ressentir aussi lorsque la boîte accueille un nouvel orchestre de jazz, celui de Léo Le Sieur et de ses Puss-in-the-Boots ou College Boys ${ }^{86}$. À l'image des petits orchestres qui font danser la jeunesse dans les hôtels, restaurants et cabarets de la métropole, l'ensemble instrumental est formé d'un piano, de trois saxophones, d'un violon et d'une batterie. Durant l'entre-deux-guerres, nombre de spectacles de ces orchestres à dancing sont quotidiennement retransmis en fin de soirée sur les ondes du poste de radio CKAC.

On comprend donc que la chanson française et l'opérette, qui faisaient la marque du café-concert montmartrois, ne sont plus vraiment en vogue. Avec ce virage vers Broadway, on se demande ce qu'il peut bien rester d'unique au Matou botté? Voyons ce qu'il en est de la danse et des sketches qui tiennent désormais l'affiche.

\section{La danse}

Comme l'illustre l'article de Peggy Roquigny dans ce numéro, la danse récréative au son d'un orchestre devient un loisir extrêmement prisé durant l'entre-deux-guerres. La réorientation du cabaret Au matou botté en nightclub de Broadway passe en partie par l'embauche de jeunes danseuses qui multiplient les engagements dans les cabarets de Montréal. À titre d'exemple, les sœurs Campbell tiennent l'affiche au Venetian Gardens, au Théâtre National et au Matou botté tout en rêvant de percer à Broadway (revoir la note 39).

${ }^{84}$ La Patrie, 13 septembre 1930, p. 20.

85 Les trois danseuses, les Goldwin Sisters et Sally Kane, forment le Varsity Trio.

${ }^{86}$ Montreal Daily Star, $1^{\text {er }}$ février 1930, p. 25; La Patrie, 5 février 1930, p. 4. Les articles mentionnent qu'Armand Meerte, Percy Sky, Wilfrid Manseau et Stuart Ellison sont des musiciens l'orchestre de Léo Le Sieur. 
Le mouvement inverse, de New York vers Montréal, est aussi observable. Par exemple, Dora Marshall annonce, en 1930, l'ouverture d'un studio de danse rue Sainte-Catherine Ouest ${ }^{87}$. La danseuse se présente ainsi : "Formerly Associate Ballet Mistress Roxy N.Y. - Ballet Mistress Capitol Theater Montreal. » Elle occupe dorénavant la scène du Théâtre Capitol avec ses Capitolettes et l'orchestre de Giuseppe Agostini (1890-1971) ${ }^{88}$. On retrouvera Dora Marshall au Matou botté en $1932^{89}$.

Un autre programme du cabaret mentionne en 1930 que Miss Sally Kane et les Goldwin Sisters sont des " danseuses de New York ${ }^{90}$ ". Leur numéro contient notamment " Le pas de l'ours " et " La danse de la couleuvre ", sans plus de détails. On peut à tout le moins émettre l'hypothèse que ces pratiques dérivent de la Roaring Twenties Dance Craze, venue des États-Unis. Cette vogue a énormément popularisé le turkey-trot, le grizzly bear, le black-bottom et surtout le fox-trot, la plus célèbre d'entre toutes ces animal dances américaines.

\section{Les sketches}

Plus que la danse et les chansons anglophones, les sketches préservent en surface l'image du café-concert montmartrois. Fin mai 1930, le numéro «Les hommes célèbres " est une adaptation fantaisiste basée sur le texte des Femmes savantes de Molière ${ }^{11}$. La semaine suivante, le chansonnier et diseur Eugène Vivez signe sa première revue d'actualité, une œuvre qui aborde pêle-mêle le passage à SaintHubert du ballon dirigeable R-100 - également immortalisé en chanson par Madame Bolduc -, la prohibition de l'alcool, la censure,

\footnotetext{
87 À l'époque, le studio de Dora Marshall est situé au 1231, rue Sainte-Catherine Ouest (voir le Montreal Daily Star, 7 février 1930, p. 6).

${ }_{88}$ Montreal Daily Star, 11 octobre 1930, p. 23.

89 La Presse, 20 août 1932, p. 44; La Presse, 27 août 1932, p. 55; La Presse, 8 septembre 1932, p. 8 ; et La Presse, 10 septembre 1932, p. 63-64.

90 La Patrie, 14 mai 1930, p. 7.

91 La Patrie, 27 mai 1930, p. 8.
} 
la mode, les taxis, la Société des Nations et les États-Unis d'Europe ${ }^{92}$. On se sent ici proche de l'esprit qui animait les revues de Bourgeois et Poirier.

Eugène Vivez présente ensuite une deuxième revue, simplement intitulée Allô 1930! En plus des habituels numéros de chant et de danse, on y remarque l'introduction sur scène d'une pantomime italienne ${ }^{93}$. Elle sera suivie d'autres scènes mimées sur "Le marchand de jouets ", "La vierge du temple " et " La bataille des fleurs ». Le premier sketch met en scène Pierrot, Arlequin et Colombine, des personnages célèbres de la commedia dell'arte. Aucun détail n'est donné sur la deuxième œuvre. Quant au dernier sketch, il présente une petite marchande de fleurs qui épouse un millionnaire, aux dépens d'un ivrogne ${ }^{94}$. La recette est entendue : des scénarios simplistes, des personnages bien campés, du burlesque avec un jeu très physique chez les acteurs, empruntant vraisemblablement au slapstick $^{95}$. L'insistance sur le jeu corporel des acteurs relègue la satire du texte français au second plan.

En ce qui concerne la présentation de pantomime, la direction s'en explique ainsi : "Pour protester contre le cinéma parlant, le Matou [botté] nous donne du théâtre muet ${ }^{96}$. II semble toutefois plus avisé de croire que le jeu muet des acteurs permet de joindre un nouvel auditoire anglophone, majoritaire au centre-ville de Montréal... et précieux en temps de crise économique. D’ailleurs, Vivez affirme sans ambages que, pour les Américains de passage, le cabaret Au matou botté, c'est Paris ${ }^{97}$. Le Montmartre du cabaret d'hier auraitil donc été transformé en un simple décor? La critique ne manque

92 La Patrie, 31 mai 1930, p. 23; La Patrie, 3 juin 1930, p. 7.

${ }^{9}$ La Patrie, 14 juin 1930, p. 22; La Patrie, 17 juin 1930, p. 8. La photo qui accompagne l'article indique clairement que Paul Max est désormais le directeur artistique des lieux.

94 La Patrie, 28 juin 1930, p. 20 ; et La Patrie, 2 juillet 1930, p. 7.

95 Voir Hébert, Le burlesque au Québec.

96 La Patrie, 3 septembre 1930, p. 5.

${ }_{97}$ La Patrie, 9 septembre 1930, p. 5. 
pas d'observer le changement d'orientation du cabaret. En juillet 1930, Noctambule - possiblement un pseudonyme d'Henri Letondal - déplore dans les pages de La Patrie que les chansons et les danses soient les mêmes depuis plusieurs mois. L'article ajoute lucidement que le programme est bien pour les Américains, mais que la clientèle de jadis n'y est plus ${ }^{98}$.

\section{Montmartre ou Broadway? Un cabaret montréalais qui se cherche...}

Fin août 1930, le directeur artistique Paul Max déclare à la presse que la politique du cabaret Au matou botté sera entièrement changée pour la nouvelle saison. En effet, il a été décidé d'engager chaque semaine un artiste local qui présentera un récital quotidien de chant ou de musique ${ }^{99}$. On admet donc à mots couverts l'échec de l'aventure Broadway des derniers mois tout en cherchant à redonner au lieu sa vocation d'origine, celle d'un café-concert de style Montmartre. Le thème du chat, les bals masqués et l'opérette seront de nouveau au menu des attractions, en plus de quelques innovations du côté du cinéma, de la danse et de la radio.

En lançant cette nouvelle année, la composition de la troupe régulière se transforme fort peu, mais elle est considérablement réduite. Max et Vivez demeurent à la direction, tandis que Léo Le Sieur continue de diriger l'orchestre. La danseuse Marguerite Bray fait toujours partie de l'équipe. Pour seule " nouveauté », on note le retour des sœurs d'Arcy, le musicien et comédien Percy Sky - déjà membre de l'orchestre de Le Sieur - de même que l'arrivée de mademoiselle Jeanne Béland.

\footnotetext{
98 La Patrie, 22 juillet 1930, p. 5.

99 La Patrie, 30 août 1930, p. 23.
} 


\section{Léo Le Sieur, nouveau maître d'œuvre}

En octobre 1930, Léo Le Sieur assure lui-même pendant un mois la direction du cabaret Au matou botté ${ }^{100}$. Au programme, les sketches "Amour en matou » et " Griserie », deux fantaisies sur un livret de Vivez et une musique de Le Sieur. Il y a aussi une saynète conçue à partir d'une chanson de l'auteur-compositeur et réalisateur Roméo Beaudry (1882-1932), intitulée "Les baisers sont les fleurs de l'amour ${ }^{101} »$. En danse, on propose des pas hawaïens, un numéro sur pointes - du ballet classique - et quelques clog dances - un type de gigue traditionnelle $\mathrm{e}^{102}$.

On signale à quatre reprises la présence d'une certaine Madame Bolduc et de son " art du piano ${ }^{103}$ ». Il est permis de se demander s'il s'agit d'une intervention Au matou botté de la célèbre auteurecompositrice de chansons originales à saveur folklorique, accompagnée du turlutage, de sa musique à bouche et d'une partie de piano. Le journal ne donne aucune précision, sinon qu'il faut absolument entendre cette « Mme Bolduc qui nous charme par son piano durant les intermissions ".

Toujours sous la direction de Le Sieur, un nouveau portraitiste semble avoir repris le rôle laissé vacant par Albéric Bourgeois. Caricaturiste de journaux à New York, à Paris et à Philadelphie, Nino

100 La Patrie, 27 septembre 1930, p. 21 ; La Patrie, 22 octobre 1930, p. 8. La presse révélera ensuite que Léo Le Sieur quitte ses fonctions au Matou botté pour devenir organiste attitré au Théâtre Capitol de Montréal.

101 La Patrie, 4 octobre 1930, p. 22; La Patrie, 8 octobre 1930, p. 5. Roméo Beaudry est le réalisateur des disques Starr de la compagnie Compo, responsable de l'enregistrement, en 1929, des premières chansons à succès de Madame Bolduc. Il est aussi le traducteur de centaines de chansons américaines, de même que l'auteur-compositeur d'une soixantaine de chansons originales. À propos des chansons de Beaudry, voir P. Bouliane, Good-bye Broadway, Hello Montréal; et La Patrie, 14 janvier 1931, p. 7.

102 Le $c l o g$ dance est un type de gigue d'origine britannique, dans le Lancashire. Les clogs désignent également des chaussures pour danser de manière percussive avec les pieds. L'équivalent québécois serait les sabots ou les souliers ferrés.

103 La Patrie, 30 septembre 1930, p. 8 ; La Patrie, 8 octobre 1930, p. 5 ; La Patrie, 4 novembre 1930, p. 8 ; La Patrie, 30 décembre 1930. 
Sparacino est de passage à Montréal alors qu'il fait un stage comme artiste invité au Manoir Richelieu de La Malbaie. En cet automne 1930, on signale sa présence au Matou botté à quelques reprises ${ }^{104}$. Quant aux nouveaux décors du cabaret, ils sont confectionnés par Roger Couillard $^{105}$.

\section{Retour aux bals masqués et à l'opérette : le premier anniversaire}

On assiste à un certain retour aux genres qui ont fait les beaux jours de l'ouverture du cabaret Au matou botté. Cette fois-ci, cependant, le bal masqué " paré et travesti du Matou botté [est présenté] à l'occasion du Hallowe'en ${ }^{106}$ ». On ne célèbre donc plus une fête d'origine chrétienne comme la Sainte-Catherine, Noël, la MiCarême ou le Mardi gras, mais bien une fête de païenne, la veille de la Toussaint. Cette même semaine, « la seule boîte montmartroise de l'Amérique » accueille Charles-Émile Brodeur et Lucienne Defrenne pour interpréter les opérettes Les Noces de Jeannette et Vision d'Orient. Chaque soir, l'opérette tient l'affiche à 20 heures, suivie du cabaret à 22 heures.

Au début de novembre 1930, un autre grand bal est donné à l'occasion de l'Armistice ${ }^{107}$. Dans La Patrie, on prend la peine de souligner la valeur artistique de la programmation : « [I]l ne faut pas considérer le spectacle du Matou Botté comme un ensemble de vagues intermèdes de dancing : c'est bel et bien un effort artistique qui mérite qu'on s'y arrête ${ }^{108}$. » Devant cette programmation à mi-chemin entre Montmartre et Broadway, il semble évident que le cabaret montréalais se cherche un second souffle : il veut préserver son identité d'origine, tout en maintenant sa rentabilité.

\footnotetext{
104 La Patrie, 4 octobre 1930, p. 22 ; et La Patrie, 8 octobre 1930, p. 5.

105 Roger Couillard a par ailleurs fabriqué des affiches promotionnelles pour la compagnie de chemin de fer Canadien Pacifique.

106 La Patrie, 29 octobre 1930, p. 5; La Presse, 31 octobre 1930, p. 77; et La Patrie, 7 novembre 1930 , p. 8.

107 La Presse, 8 novembre 1930, p. 63.

108 La Patrie, 8 novembre 1930, p. 23.
} 
Le 26 novembre 1930, le cabaret Au matou botté souligne son premier anniversaire avec un nouveau grand bal de la Sainte-Catherine ${ }^{109}$. Dans La Patrie, un communiqué vante l'image du café-concert montmartrois des premiers jours :

Comme tous le savent, c'est cette semaine que le Matou botté, ce cabaret de la rue Saint-Denis, qui est si populaire et où l'on retrouve de façon incontestable l'atmosphère parisienne du vieux cabaret montmartrois, célèbre son premier anniversaire de fondation. Oui, c'est il y a un an que ce bon vieux Matou a chaussé ses bottes pour la première fois. Son succès depuis ne s'est pas départi ${ }^{110}$.

Au programme, on a invité le monologuiste Jules Ferland à revenir donner un spectacle, le temps d'une soirée. Il partage la scène avec les directeurs Paul Max et Eugène Vivez, la jeune Jeanne Béland et les danseuses. Les directeurs fondateurs Roméo Poirier et Albéric Bourgeois, eux, brillent par leur absence...

\section{Quelque chose ne va pas}

En décembre 1930, le cabaret Au matou botté inaugure un « arrangement spécial » avec le Thêâtre Capitol, où travaille désormais Léo Le Sieur. Voici ce que rapporte La Patrie:

Le populaire cabaret français, le Matou botté, après arrangement spécial avec le Théâtre Capitol recevra, chaque semaine, comme ses invités de la Boîte, les vedettes de cinéma de passage à Montréal. La semaine dernière, c'était [l'actrice et chanteuse de Broadway] Lillian Roth. C'est maintenant [l'actrice canadienne-française] Fifi d'Orsay ${ }^{111}$.

109 La Presse, 22 novembre 1930, p. 64.

110 La Patrie, 26 novembre 1930, p. 9.

111 La Patrie, 9 décembre 1930, p. 8. 
Ce partenariat entre le Théâtre Capitol et le cabaret sera sans doute de courte durée, car les communiqués n'en referont pas mention. Et de nouveau, un réveillon de Noël vient clore l'automne ${ }^{112}$.

À partir de 1931, les articles au sujet du cabaret Au matou botté se font extrêmement rares dans les journaux. Les quelques textes retrouvés cachent difficilement le fait que les affaires ne tournent pas rond. À la fin janvier 1931, on peut lire dans La Patrie :

[Après le] brouhaha et l'affluence de la période des fêtes [...] Max et Vivez ont appelé à leur aide des troupes fraîches, du matériel plus solide et quelques surprises inédites pour affronter l'adversaire difficile qu'est le public de cabaret et la " critique ${ }^{113}$.

En février 1931, on organise une soirée du Mardi gras avec Armand Meerte, musicien de l'orchestre de Léo Le Sieur ${ }^{114}$. Il faut ensuite attendre deux mois avant que ne paraisse l'annonce d'un programme dans les journaux ${ }^{115}$. Paul Max, Eugène Vivez et Armand Meerte accueillent alors de nouvelles danseuses, présentées sous le nom des " 4 Matouses Girls ", à la façon d'un nightclub de Broadway.

En avril 1931, le journal La Patrie annonce que l'on fêtera bientôt " le deuxième anniversaire de la populaire boîte ${ }^{116}$ »! Moins de cinq mois après le premier anniversaire, célébré en novembre 1930, on cherche donc à créer artificiellement un événement pour attirer les spectateurs. À compter de mai 1931, la presse ne signale plus rien, ce qui donne un temps l'impression que le cabaret Au matou botté a définitivement cessé ses activités. Puis, fin novembre 1931, une publicité du restaurant Kerhulu \& Odiau annonce discrètement qu'un certain monsieur Mignolet en est le nouveau gérant ${ }^{117}$. On parle alors de dîner dansant, mais aucune mention du Matou botté

112 La Patrie, 20 décembre 1930, p. 21.

113 La Patrie, 24 janvier 1931, p. 22.

114 La Patrie, 14 février 1931, p. 22.

115 La Patrie, 11 avril 1931, p. 21.

116 La Patrie, 25 avril 1931, p. 21.

117 La Presse, 21 novembre 1931, p. 66. 
n'accompagne la publicité. La couverture de presse est la même pour le dîner de Noël 1931, tenu à la Salle des Châteaux du restaurant Kerhulu \& Odiau ${ }^{118}$. Nous sommes précisément au lieu du cabaret Au matou botté, mais sans que la réclame n'en dise un seul mot.

Dans la même page de ce journal, une autre publicité annonçant les spectacles à l'affiche nous apprend qu'Eugène Vivez et ses ballerines travaillent désormais aux côtés de Juliette Béliveau (1889-1975), au Théâtre National. Deux autres publicités du restaurant Kerhulu \& Odiau, sans aucune mention du Matou botté, confirment ce qui se présente comme une fermeture du cabaret montmartrois ${ }^{119}$. Et pourtant...

\section{La réouverture du cabaret}

En mars 1932, une nouvelle publicité du restaurant Kerhulu \& Odiau annonce en grande pompe l'« ouverture du Nouveau Salon Parisien Au matou botté [avec une] décoration nouvelle ${ }^{120}$ ", sous la direction du propriétaire Jos. Mignolet. Un communiqué publié quelques jours plus tard donne davantage de détails :

La petite boîte parisienne chez Kerhulu et Odiau, rue Saint-Denis, fermée en novembre dernier, a rouvert ses portes samedi soir. [...] Si on regrette quelque peu son ancienne parenté avec les boîtes montmartroises, on ne peut qu'admirer son rapprochement au genre des « salons " parisiens. La salle a été repeinte et re-décorée. Tout au fond se trouve encore l'énorme tête de chat qui regarde d'un oil paternel les évolutions des danseurs ${ }^{121}$.

En avril 1932, le cabaret Au matou botté inaugure une nouvelle série de soirées de gala du mercredi soir, avec en vedette Eugène Vivez, pensionnaire du Théâtre National ${ }^{122}$. On ajoute que messieurs

\footnotetext{
118 La Presse, 19 décembre 1931, p. 65.

119 La Presse, 23 décembre 1931, p. 8; et La Presse, 31 décembre 1931, p. 10.

120 La Presse, 10 mars 1932, p. 10.

121 La Presse, 15 mars 1932, p. 12.

122 La Presse, 7 avril 1932, p. 12.
} 
Mignolet et Jouglet cherchent à donner à la boîte l'aspect d'un dancing parisien, avec javas, tangos et biguines. Il semble que la concurrence flaire immédiatement la bonne affaire : moins de deux semaines plus tard, une publicité annonce l'ouverture du nouveau cabaret Chez Maurice, une salle de danse récréative à la française, sise dans l'ancienne salle du Venetian Gardens ${ }^{123}$.

Du 9 au 13 mai 1932, le cabaret Au matou botté lance une nouvelle initiative afin de se distinguer de ses compétiteurs : une semaine du film parlant français, organisée par la Compagnie cinématographique canadienne limitée de la rue Bleury ${ }^{124}$. Voilà un bien curieux retour du pendule pour le cabaret qui a jadis mis à l'affiche des pantomimes italiennes afin de lutter contre le cinéma parlant! Le communiqué se lit comme suit :

M. H. de Lanauze, distributeur des appareils Pathé-Baby $(\mathrm{m} / \mathrm{m}$ 9.5) et le fantaisiste Eugène Vivez, directeur artistique du «MATOU BOTTÉ » chez Kerhulu \& Odiau, organisent en cet endroit un concours cinématographique qui commencera lundi soir à 11 heures et durera jusqu’à samedi soir.

L'article précise qu'Eugène Vivez filmera de courtes prises de vues de trois dames. Ces films seront ensuite projetés devant le public. L'opération est appelée à se répéter tous les soirs de la semaine. Un concours couronnant la Montréalaise la plus photogénique doit clore le tout. Cette expérience cinématographique ne semble pas s'être répétée par la suite. Au début de juin 1932, quelques nouvelles publicités font la promotion du restaurant Kerhulu \& Odiau, encore une fois sans mentionner le cabaret Au matou botté ${ }^{125}$.

${ }^{123}$ La Patrie, 23 avril 1932, p. 18. Chez Maurice loge au 1224, rue Sainte-Catherine Ouest, selon la numérotation civique de l'époque.

124 La Patrie, 7 mai 1932, p. 12; et La Presse, 7 mai 1932, p. 64-65.

125 La Presse, 8 juin 1932, p. 8 ; La Patrie, 8 juin 1932, p. 7 ; et La Presse, 9 juin 1932, p. 12. 


\section{Dernières tentatives : la danse et la radio}

Pendant l'été 1932, le cabaret tente une relance sous le signe des danses internationales, avec Laura Reld et Miggy du Théâtre Roxy de New York ${ }^{126}$. Le spectacle prend la forme d'une revue des grandes danses nationales : le tango d'Argentine, la rumba de La Havane (à Cuba), la valse de Vienne (en Autriche), le menuet de France et un peu plus énigmatique - la danse acrobatique de Suède. La petite troupe régulière du cabaret se compose maintenant d'Eugène Vivez à la direction, de Lou Gravel à l'orchestre et de la soprano Jeanne Mignolet - possiblement la fille du nouveau propriétaire.

Au début de septembre 1932, une nouvelle revue intitulée Un voyage en Chine partage l'affiche avec Jeanne Mignolet, Georges Dufresne et les danseuses de Dora Marshall ${ }^{127}$. Plus que jamais auparavant, le cabaret montréalais voit grand en cherchant à s'ouvrir sur le monde entier.

Le cabaret annonce peu après qu'il aura sa propre émission de radio à CKAC, la Revue du Matou botté ${ }^{128}$. Le communiqué de La Presse en fait la description suivante :

Ceci est le premier d'une série de petits concerts hebdomadaires que le Matou botté, le gai cabaret français à l'enseigne de Kerhulu et Odiau, 1284, rue Saint-Denis, donne tous les lundis [de $18 \mathrm{~h} 15$ à 18 h 30, à compter du 19 septembre 1932]. On y entendra les grandes vedettes prenant part au spectacle de la semaine courante et l'excellent orchestre de danse du Matou donnant des extraits des primeurs qu'il jouera de temps à autre ${ }^{129}$.

Lémission paraît chaque semaine dans la grille-horaire de CKAC jusqu'au 22 octobre $1932^{130}$. La semaine suivante, on organise une

${ }^{126}$ La Presse, 23 juillet 1932, p. 45; La Presse, 3 août 1932, p. 8 ; et La Presse, 6 août 1932, p. 55.

127 La Presse, 10 septembre 1932, p. 63-64; et La Presse, 13 septembre 1932, p. 8.

128 La Patrie, 17 septembre 1932, p. 17.

129 La Presse, 19 septembre 1932, p. 18.

130 La Patrie, 24 septembre 1932, p. 32; La Patrie, $1^{\text {er }}$ octobre 1932, p. 17 ; La Patrie, 8 octobre 1932, p. 17; et La Patrie, 22 octobre 1932, p. 17. 
fête chez Kerhulu \& Odiau à l'occasion de l'Halloween, encore une fois sans aucune allusion au Matou botté ${ }^{131}$. Ce serait donc vraiment la fin des activités artistiques de la "boîte montmartroise " à Montréal.

Plusieurs des anciennes têtes d'affiche du cabaret assureront une présence à la radio dans les années 1930. En 1933-1934, Roméo Poirier sera le maître de cérémonie du Radio-divertissement Molson, émission commanditée par la brasserie, qui existe toujours. Il partage la vedette avec le chef d'orchestre Giuseppe Agostini et l'acteur Hector Charland, dans le rôle du père Molson. Au début de 1936, Albéric Bourgeois animera le Programme Alouette, sous les auspices de la compagnie de tabac B. Houde Limitée ${ }^{132}$. Il signera, dans le cadre de cette émission, quelques centaines de chansons d'actualité sur timbres. Enfin, Léo Le Sieur dirigera, à l'automne 1936, l'émission de chansons Sweet Caporal, une compagnie de tabac bien connue à l'époque.

\section{Conclusion}

En 1929, le cabaret dirigé par Albéric Bourgeois et Roméo Poirier réussit à se démarquer de la concurrence des théâtres anglais en affichant clairement son identité de café-concert à la mode de Montmartre. Sous le signe du Chat noir, la programmation de chansons, d'opérettes, de sketches et de thés dansants chez Kerhulu $\&$ Odiau devient vite un attrait prisé de la bourgeoisie canadiennefrançaise. Quelques mois plus tard, lorsque les Eugène Vivez et Paul Max reprennent l'établissement, le choix le plus rentable est celui d'une programmation alignée sur le modèle du nightclub de Broadway. Les danseuses et la pantomime alternent avec le burlesque et le jazz, mais la clientèle des débuts déserte le lieu. En dépit des

131 La Presse, 28-29 octobre 1932, p. 8.

132 Un recueil de chansons issu de cette émission est d'ailleurs conservé à BAnQ. Voir Albéric Bourgeois, Joson et Josette au micro : chansons du programme, [1936], Québec, Compagnie B. Houde ltée. 
expériences avec le cinéma et la radio, cette tension constante entre les pratiques de Montmartre et celles de Broadway apparaît, avec le recul, comme le talon d'Achille du cabaret. C'est peut-être finalement Madame Bolduc qui aura le mieux résolu la dialectique entre identité et rentabilité, dans son cas grâce au disque et à une troupe de variété itinérante sillonnant le Québec et le nord-est des États-Unis.

On peut se demander si le café-concert Au matou botté aurait mieux tiré son épingle du jeu en s'inspirant différemment du folklore. Bourgeois et Poirier l'ont fait au moins une fois à partir d'un tableau de l'illustrateur Edmond-Joseph Massicotte. Une recette somme toute analogue a aussi assuré pendant une vingtaine d'années le succès des Veillées du bon vieux temps de Conrad Gauthier, au MonumentNational. Rappelons que c'est justement dans le contexte de ces veillées, en 1928, que l'on découvre la chansonnière turluteuse de reels.

Le cas du cabaret Au matou botté donne néanmoins à voir que les artistes chanteurs, auteurs de sketches et danseuses se préoccupent de ce qui se fait en leur temps à Paris et à New York. Cette ouverture sur les deux grandes métropoles culturelles d'Europe et d'Amérique s'inscrit en porte-à-faux par rapport au Canada français replié sur lui-même, comme on l'imagine souvent en pensant à cette époque. Le parallèle m'apparaît en cela frappant avec la programmation générale du poste de radio CKAC, officiellement de langue française le seul en Amérique avant 1930 -, mais largement bilingue dans les faits, et très ouvert sur la musique classique européenne de même que sur la musique populaire ou le jazz américain.

On s'étonne enfin de la présence de "chansonniers " dans la programmation du cabaret Au matou botté, dès 1929, au moment précis du premier succès sur disque de Madame Bolduc. Albéric Bourgeois et Gilles-René Amiot, pour ne nommer qu'eux, s’affichent en effet comme " chansonniers " d'une boîte montmartroise, et ce, plus de vingt ans avant l'arrivée de Félix Leclerc à Paris. Le sens attribué au mot " chansonnier " est important. Tandis que Bourgeois et Amiot sont des paroliers de chansons d'actualité sur des airs connus, 
Madame Bolduc va s'affirmer dès le début comme auteurecompositrice-interprète. La Revue populaire de mars 1931 la présente d'ailleurs clairement comme " chansonnière [...] qui compose ellemême la musique et le texte de toutes ses créations ${ }^{133}$ ".

Il y aurait lieu d'examiner plus scrupuleusement la façon dont se met en place ce " chansonnier " auteur-compositeur au Québec entre 1900 et 1960, à l'Eldorado, au Faisan Doré et à la ComédieCanadienne de Gratien Gélinas, par exemple. Plus généralement, le retour aux archives de la presse écrite nous invite à repenser l'esthétique des boîtes à chansons dans leur opposition aux groupes de rock and roll ou yé-yé. On peut légitimement se demander s'il y aurait eu en ces lieux un métissage des pratiques de la chanson plus grand que ce que l'histoire retient en général.

133 « Madame Bolduc : chansonnière populaire », La Revue populaire, mars 1931, p. 15 . 